\title{
Essays in games and decisions
}

Citation for published version (APA):

Ismail, M. S. (2017). Essays in games and decisions. [Doctoral Thesis, Maastricht University]. Off Page Amsterdam. https://doi.org/10.26481/dis.20170608msi

Document status and date:

Published: 01/01/2017

DOI:

10.26481/dis.20170608msi

Document Version:

Publisher's PDF, also known as Version of record

\section{Please check the document version of this publication:}

- A submitted manuscript is the version of the article upon submission and before peer-review. There can be important differences between the submitted version and the official published version of record.

People interested in the research are advised to contact the author for the final version of the publication, or visit the DOI to the publisher's website.

- The final author version and the galley proof are versions of the publication after peer review.

- The final published version features the final layout of the paper including the volume, issue and page numbers.

Link to publication

\footnotetext{
General rights rights.

- You may freely distribute the URL identifying the publication in the public portal. please follow below link for the End User Agreement:

www.umlib.nl/taverne-license

Take down policy

If you believe that this document breaches copyright please contact us at:

repository@maastrichtuniversity.nl

providing details and we will investigate your claim.
}

Copyright and moral rights for the publications made accessible in the public portal are retained by the authors and/or other copyright owners and it is a condition of accessing publications that users recognise and abide by the legal requirements associated with these

- Users may download and print one copy of any publication from the public portal for the purpose of private study or research.

- You may not further distribute the material or use it for any profit-making activity or commercial gain

If the publication is distributed under the terms of Article $25 \mathrm{fa}$ of the Dutch Copyright Act, indicated by the "Taverne" license above, 
Essays in Games and Decisions

Mehmet S. Ismail 
Essays in Games and Decisions

(C) Mehmet S. Ismail, Maastricht 2017

All rights reserved. No part of this publication may be reproduced, stored in a retrieval system, or transmitted, in any form, or by any means, electronic, mechanical, photocopying, recording or otherwise, without the prior permission in writing from the author.

This book was typeset by the author using $\mathrm{HT}_{\mathrm{E}} \mathrm{X}$.

ISBN 978-94-6182-803-3

Printed in the Netherlands by Off Page 


\section{Essays in Games and Decisions}

\section{DISSERTATION}

to obtain the degree of Doctor at Maastricht University, on the authority of the Rector Magnificus,

Prof.dr. Rianne M. Letschert

in accordance with the decision of the Board of Deans, to be defended in public

on Thursday 8 June 2017, at 12:00 hours

by

Mehmet S. Ismail 


\section{Supervisor:}

Prof.dr. J. J. Herings

\section{Co-Supervisor:}

Dr. R. Peeters

\section{Assessment Committee:}

Prof.dr. H. J. M. Peters (chair)

Dr. P. Duersch, Heidelberg University, Germany

Prof.dr. B. C. Schipper, University of California, Davis, United States of America

Dr. A. Predtetchinski 


\section{Acknowledgements}

First and foremost, I would like to thank my supervisors, Ronald Peeters and Jean-Jacques Herings. Ronald, you have been not only an excellent supervisor, but also a friend. I have enjoyed both our academic and non-academic talks which ranged from politics to religion. You inspired me with your genuine interest in helping, and-as a theorist and an experimentalist-you have asked challenging questions and encouraged me during my studies. The things you have done for me will not be forgotten. Jean-Jacques, you have always been available whenever I needed the most help. Your extensive feedback for my papers was crucial: I knew that, even if I missed something, you would not. I am extremely lucky to have had you both as my supervisors. I would not be able to complete this dissertation without your support and help.

I would like to express my sincerest gratitude to Steven Brams, with whom I met at the 2014 Stony Brook Game Theory Conference. I cannot succinctly list all the things I have learned from you, but I can say without hesitation that visiting you at the 2015 spring semester at New York University was a turning point in my PhD. Our collaboration has significantly changed and shaped my views on research and life. It is perhaps ironic that our less academic interests in playable games eventually led to fruitful academic collaborations with Aaron Isaksen, Andy Nealen, Marc Kilgour, and Walter Stromquist. As the least experienced one in the group, it was a challenge to keep up with you. Thanks, Aaron, I hope 
that academia can keep a great talent like you, but, not matter our next steps, I hope that our paths will cross again. Also, thanks, Andy, it was nice to meet you (and Frank Lantz) at the Game Center at NYU.

In 2016, I met Marc and Walter in person at the Dagstuhl Seminar: Fair Division, which was the most cooperative academic event that I have ever attended. Thank you, Marc, for our collaboration; it was a nice coincidence that the author of one of my favorite books in game theory (Two-Person Game Theory) turned out to be your PhD supervisor. Thank you, Walter, I truly enjoyed our conversations, including your memorable illustration of how to divide a bottle of wine fairly among three persons, and, of course, the backgammon game that we played! I also want to extend my thanks to Yonatan Aumann (winner of the first mini Catch-Up tournament), Jérôme Lang, Ariel D. Procaccia (organizers), Edith Elkind, Hervé Moulin, Marco Dall'Aglio, Yann Chevaleyre, Avinatan Hassidim, Katarina Cechlarova, Michael Jones, and Erel Segal-Halevi (a nice friend with whom we played catch up games together) for interesting talks and discussions.

I was fortunate to have met Nejat Anbarc1 at the 5th Game Theory Congress at Maastricht University. I enjoyed our long conversations into the night. I would like to thank Kutay (especially for the great memories that we have shared in Maastricht) and you for our collaboration. I also would like to thank Tigran Melkonyan, whom I visited for a month at Warwick Business School. You were so friendly and kind that you shared your office with me during my visit. I enjoyed our behavioral group meetings and e-mail discussions with Nick Chater, Jennifer Misyak, and Hossam Zeitoun; I am thankful to you all.

There is also specific gratitude behind each of the chapters of this dissertation. The first paper (Chapter 2) of my dissertation started as a term paper in a Decision Theory course with Itzhak Gilboa. I am grateful for your comments especially on the first version and found your classes 
inspirational (e.g., lemon markets, my name is red). Incidentally, this paper evolved into my master's thesis which was supervised by Philippe Bich and Frank Riedel, and which ultimately led to the second and third chapters of this dissertation. I learned a lot in Fixed Points Theory and Functional Analysis courses with Philippe Bich; I am grateful to you as those courses helped me prove several theorems in this thesis. Further, Chapters 4 and 5 were both born out of my explorations of the preceding two chapters. I started Chapter 5 during my visit at the Department of Politics at New York University. I am grateful to the Department for its hospitality during my visit, which was possible thanks to the GSBE at Maastricht University. I also want to thank, Elke Lucas, Nicole Hulsman, and Sylvia Beenen, for their administrative help during my $\mathrm{PhD}$, and Arno Riedl for nice dinners; and Esther Kockelkoren, Ivo George, Margot Krijnen, and Robert Fechter for their efforts on copyrighting and/or publicizing the Catch-Up Game and Rule.

I was inspired by and profited from many personal discussions with Andrés Perea, János Flesch, Hans Peters, Dries Vermuelen, Matthew Embrey, Christian Bach, Elias Tsakas, Kristof Bosmans, Martin Strobel, Philippe Bich, Karl Schlag, Peter Wakker, Frank Riedel, Francis Bloch, Willemien Kets, and Marcus Pivato. You may not realize how motivating these conversations have been during this process. I also would like to thank my professors, from whom I learned a lot about Economics and Mathematics, including Jean-Bernard Baillon (for Hilbert spaces, CauchySchwarz inequality, great inspiring lectures, and "we found it!"), Alain Chateauneuf, Mohammed Abdellaoui, Bernard Cornet (especially for his proof techniques), Jean-Marc Bonnisseau, Christoph Kuzmics, Pascal Gourdel, Bernard de Meyer, Joseph Abdou, Agnieszka Rusinowska, Volker Böhm (for the inspiring question: What's the difference between the forecasts of a weather forecaster and an economist?), and Julien Randon-Furling. 
Acknowledgments

Wherever I have been in my life, I have been extremely lucky to have been surrounded by beautiful friends. These friendships, new and old, have also been vital to the ups and downs of PhD life. Next I would like to extend my thanks to them.

My years at Maastricht would not be as enjoyable without many PhD activities, barbecue days, sushi (Tokyoto), and carnival, some of which I enjoyed with Murat, Çiğdem, Burcu, Hande, Emel, Demet, Livy, Ayşe, Kutay, Anna, Merve, Esin, Amber, Deniz, Sevim, Esra, Elvin, Gabri, Aline, Anne, Vera, Adam, Firat, Aidas, Alexander, Yeliz, Arkadi, Birol, Erkan, Elçin, Henrik, Riccardo, Jasmine, Nickolas, Marcello, Anastas, and Maria. I am also thankful to the friends with whom I have shared office including Evi (parakalo), Özge and Devrim (Donderdags), Christine, Hande, Florian, Seher, Merve, Norman, and of course Diogo (with whom we have shared countless memories together since the first year of my $\mathrm{PhD}$, merci, mon ami!).

I met some fantastic people during and after my QEM master's in Paris and Bielefeld including Lore (we go to BnF!?), Vitto, Barış (guys, I am sure we will unanimously agree on the Monty Hall problem some day!), and Diane, Ale, Hamzeh, Manue, Giulia, Joanna, Lenka, Kuba, Aleksandra, Paula, Stefanija, Can, Isacco, Francesco, and Giorgio. I shared great memories with Demet, Sinem, Göznur, Leyla, Asuman, Abishek, Metin, Tuğba, İdil, Andrea, Anastasia, Binghuan, Wei, Kuba, Ido, Helen, Qing, Sandra, Stephanie, Olga, and of course Ziad! Special thanks go to my paranymphs, Vitto and Devrim, who will be coming all the way from Milan and Istanbul, respectively, and Evi and Diogo for their help with my defense preparations.

Part of the reason I work with games is because I love playing games. I played chess at the clubs at Naval High School, YTU, and the Sorbonne, and backgammon at the Istanbul Backgammon Club (mostly), New York City, and Coventry; I thank my fellow members for great games and the viii 
Acknowledgments

moments we enjoyed together. I also would like to thank TTV Maastricht for creating a friendly atmosphere to play table tennis and their members with whom I enjoyed playing including Bob and Stan (dank je wel)! I also would like to thank Kelly for proofreading my thesis.

I shared unforgettable memories with my friends, whom I met at Yıldız Technical University and elsewhere, including Özalp, Elif, Adnan, Ekin, Sonay, Burak, Barış, Duygu, Dilek, Firdevs, Mehmet, Burak, Emre, Yasemin, and Ayazağa Tayfası. I am grateful to my professors Feride Gönel, Ensar Y1lmaz, and Nuri Yıldırım, who supported me to pursue an academic career, and Ömer Dinç for his kindness, inspiration, and for believing in me.

Last, but not least, I am grateful to my parents for their unconditional love and support, and my dear sister, Vildan, for always being so kind to me.

Mehmet S. Ismail

Maastricht, May 3, 2017 



\section{Contents}

Acknowledgements v v

1 Introduction 1

2 Two-person symmetric games and decision problems $\quad 7$

2.1 Introduction . . . . . . . . . . . . 7

2.2 The framework . . . . . . . . . . . . . . . 9

2.3 Games in pure strategies and decision problems in a riskless context . . . . . . . . . . . . . . . 11

2.3.1 The equivalence mapping . . . . . . . . . . 11

2.3.2 Counterparts of maximal elements . . . . . . . . 13

2.4 Mixed extension of games and decision problems . . . . 15

2.4.1 Two-player symmetric zero-sum games . . . . . . 15

2.4 .2 Two-player symmetric games . . . . . . . . . . . 19

2.5 The independence axiom and potential games . . . . . 21

2.6 Conclusion . . . . . . . . . . . . . . . . . 28

3 Existence of pure equilibrium in two-person symmetric zerosum games 29

3.1 Introduction . . . . . . . . . . . . . . . . . . . 29

3.2 The framework . . . . . . . . . . . . 30

3.3 Sufficient conditions . . . . . . . . . . . . . 31

3.4 Relations between sufficient conditions . . . . . . . . 34 
3.5 Examples . . . . . . . . . . . . . . . . . 38

3.6 Conclusion . . . . . . . . . . . . . . . . . 40

4 Optimin equilibrium: An extension of maximin strategies 43

4.1 Introduction . . . . . . . . . . . . . . . . . 43

4.2 Definition of optimin equilibrium . . . . . . . . . 47

4.2.1 Existence of optimin equilibrium . . . . . . . . . 53

4.3 Properties and more examples . . . . . . . . . . . . 55

4.4 An application: Arrow-Debreu economy . . . . . . . . 60

4.5 Conclusion . . . . . . . . . . . . . . . . 62

5 Is rationality a personal trait? A paradox 65

5.1 Introduction and setting $\ldots \ldots \ldots \ldots$

5.2 An example and conclusion . . . . . . . . . . . 69

$\begin{array}{ll}\text { Bibliography } & 77\end{array}$

$\begin{array}{ll}\text { Valorization } & 79\end{array}$

$\begin{array}{ll}\text { Biography } & 85\end{array}$ 


\section{Chapter 1}

\section{Introduction}

Luce and Raiffa (1957) co-authored one of the first books about game theory, entitled "Games and Decisions: Introduction and Critical Survey," a title suggested by A. W. Tucker. This book inspired the title of the current dissertation in which I explore some interrelations between games and decision problems.

In a decision problem, an agent makes a choice from a set of alternatives whose outcomes are usually unknown. If the probabilities in the uncertain situation are known to the agent, the uncertainty is called risk; if the probabilities are unknown, it is called Knightian uncertainty or ambiguity. A strategic game (say, in normal-form) can be viewed as another type of uncertainty in which outcomes depend on the choices of players who do not possess any information about the choices of others. However, unlike ambiguity, strategic uncertainty is controlled by players themselves-who may have conflicting interests over the outcomesrather than an exogenous process (e.g., drawing a ball from an unknown urn).

In this dissertation, I will depict certain unexplored relations between uncertainties in games and decision problems. However, I do not take a side in the discussion of whether strategic games should be treated 


\begin{tabular}{c|c|c|c|}
\multicolumn{1}{c}{} & \multicolumn{1}{c}{$\mathrm{R}$} & \multicolumn{1}{c}{$\mathrm{S}$} & \multicolumn{1}{c}{$\mathrm{P}$} \\
\cline { 2 - 4 } $\mathrm{R}$ & 0,0 & $1,-1$ & $-1,1$ \\
\cline { 2 - 4 } $\mathrm{S}$ & $-1,1$ & 0,0 & $1,-1$ \\
\cline { 2 - 4 } $\mathrm{P}$ & $1,-1$ & $-1,1$ & 0,0 \\
\cline { 2 - 4 } & &
\end{tabular}

Figure 1.1: Rock-Paper-Scissors game.

as multi-person decision problems, or decision problems as one-person games. Moreover, I will propose an alternative solution that arguably provides novel insight into some non-cooperative games.

\section{Outline of Chapters 2-5}

\section{Chapter 2}

In this chapter, I will define a simple equivalence relation between the class of two-person symmetric games and the class of decision problems with a complete but not necessarily transitive preference relation. To illustrate this equivalence and show why transitivity is not assumed, consider the Rock-Paper-Scissors game in Figure 1.1, which is known by its cyclic structure (recall that rock beats scissors, scissors beats paper, and paper beats rock). The equivalent decision problem of this game is defined as the pair, $(X, \succeq)$, in which $X=\{R, P, S\}$ and $R \succ S \succ P \succ R$. In other words, the decision maker's preference relation over the choices is cyclic.

Under this equivalence, I will highlight the links between some game and decision theoretical concepts. For example, a Nash equilibrium in a two-person symmetric zero-sum game and a pair of maximal elements in its equivalent decision problem coincide. Further, I will show that a twoperson symmetric zero-sum game can be extended to its von NeumannMorgenstern (vN-M) mixed extension if and only if the extended decision problem satisfies SSB utility (Fishburn, 1982), which is a weaker notion 
than vN-M utility.

I will also demonstrate that a decision problem satisfies vN-M utility if and only if its equivalent symmetric game is a potential game (Monderer and Shapley, 1996). Accordingly, I will then provide a formula as a function of number of strategies for the number of linearly independent equations that must be satisfied for a potential function to exist in these games. The value of the formula, $\frac{(n-1)(n-2)}{2}$, grows quadratically as the number of strategies $(n)$ increase.

\section{Chapter 3}

In this chapter, I will introduce three sufficient conditions for the existence of a pure Nash equilibrium in two-person symmetric zero-sum games: (1) a quasiconcavity notion based on the preferences of a decision maker in the equivalent decision problem of a game; (2) a functional representation of an axiom in Fishburn and Rosenthal (1986); (3) the so-called sign-quasiconcavity notion, which is a generalization of quasiconcavity in Duersch et al. (2012a).

I will show how these conditions are related to each other and other established sufficient conditions in the literature. The most general condition is sign-quasiconcavity which generalizes (1), (2), generalized ordinal potentials, and quasiconcave games. I will also prove that if a twoperson symmetric zero-sum game has a generalized ordinal potential, then the preference relation in its equivalent decision problem must be transitive.

\section{Chapter 4}

Because economic interactions are not typically zero-sum, maximin strategies cannot capture mutual gains and losses in these interactive situations. Thus, in Chapter 4, I will introduce a new concept, optimin equilibrium, that extends the maximin strategy solution to nonstrictly 
competitive games by incorporating an individual rationality constraint whereby players do not harm themselves for the sake of harming others.

I will show that optimin equilibrium consists of a pair of maximin strategies in zero-sum games, but this is not necessarily the case in nonzero-sum games. This result implies that the optimin equilibrium, the minimax solution, and the Nash equilibrium coincide at the mixed strategy profile $\left[\left(\frac{1}{3}, \frac{1}{3}, \frac{1}{3}\right),\left(\frac{1}{3}, \frac{1}{3}, \frac{1}{3}\right)\right]$ in the Rock-Paper-Scissors game illustrated in Figure 1.1.

I will also prove that every finite game in mixed extension possesses an optimin equilibrium, which may or may not be Pareto optimal. As an example, optimin equilibrium selects the two Pareto optimal profiles, (Football, Football) and (Opera, Opera), in the battle of the sexes game. However, it may select a Pareto dominated solution such as (Defect, Defect) in the prisoner's dilemma. Nevertheless, optimin equilibrium and Nash equilibrium may differ in the finitely repeated version in which the Tit-for-Tat strategy profile-whereby a player begins by cooperating in the first round and then does what the opponent did in the previous round-turns out to be an optimin equilibrium. For another example, consider the following one-shot game with ordinal payoffs.

\begin{tabular}{r|c|c|c|}
\multicolumn{1}{c}{} & \multicolumn{1}{c}{ Top } & \multicolumn{1}{c}{ Middle } & \multicolumn{1}{c}{ Bottom } \\
\cline { 2 - 4 } Top & 100,100 & 100,105 & 0,0 \\
\cline { 2 - 4 } Middle & 105,100 & 95,95 & 0,210 \\
\cline { 2 - 4 } Bottom & 0,0 & 210,0 & 5,5 \\
\cline { 2 - 4 } & & &
\end{tabular}

In this game, the unique Nash equilibrium is (Bottom, Bottom), whereas the unique optimin equilibrium is (Top, Top) because each player guarantees the highest payoff (100) at this profile under any profitable deviation of the other player. For example, Player 2 might profitably deviate from (Top, Top) to Middle, but Player 1 would still receive 100 in this case. However, the maximin strategy concept says that Player 2 would deviate to any feasible strategy including Bottom, leaving Player 
1 with a payoff of 0 ; though, this deviation is not in the self-interest of the deviator because the deviator also receives 0 . Thus, the individual rationality constraint of a player rules out such deviations.

Finally, I will apply this concept to the Arrow-Debreu economy and provide some sufficient conditions for its existence. My analysis will show that optimin equilibrium generalizes the concept of competitive equilibrium.

\section{Chapter 5}

In this chapter, I will focus on the concept of rationality (à la von NeumannMorgenstern) to answer the following question: 'Is it possible for an economic agent to be simultaneously rational in every situation she faces?' I will show that there are games and decision problems in which an agent cannot be simultaneously rational in both. Put differently, the same person can be simultaneously rational and irrational, hence the paradox.

Player 1's associated decision problem $D$ of a two-person game $G$ is defined as follows: (1) Player 1's monetary payoffs in $D$ are identical to those in $G$; (2) there is no payoff for another player in $D$ in which an unconscious lottery-instead of Player 2-chooses actions. (For simplicity, I assume that all outcomes are monetary.) For example, suppose that $c_{1}$ is a consequence in a game in which Player 1 receives 5 Euros and Player 2 receives 10 Euros. Then, $c_{1}^{\prime}$ is the consequence in its associated decision problem in which Player 1 receives 5 Euros:

$$
c_{1}=(€ 5, € 10) \rightarrow c_{1}^{\prime}=€ 5 .
$$

Because Player 1's monetary payoffs in the game are completely identical to her monetary payoffs in the associated decision problem, the difference in her preferences (if any) come from the fact that she plays the game against a human player who also receives payoffs. For example, if Player 1 receives less utility from $c_{1}$ than $c_{1}^{\prime}$, it means that she 
is "unhappy" about Player 2 receiving $€ 10$.

Naturally, one would like to assume that the player is rational both in the game and the decision problem. However, it turns out that Player 1 's rationality in a domain does not necessarily imply her rationality in the other domain, which may challenge the belief that rationality is a personal trait.

I will describe a necessary and sufficient condition called doubly linearity for a decision maker that makes her simultaneously rational in both situations. I will show that if a player is rational in a game, then she cannot be rational in the associated decision problem unless her social preference function satisfies doubly linearity. This condition seems to rule out social preferences including inequality aversion and altruism. 


\section{Chapter 2}

\section{Two-person symmetric games and decision problems}

\subsection{Introduction}

Rosenthal (1981) argues that games should be treated as one-person decision problems, whereas Fudenberg and Levine (2006) argue that some decision problems can be treated as a game between the different selves of a decision maker. In this chapter, I will portray symmetric games as decision problems and decision problems as symmetric games. Accordingly, I will show some equivalence relations between established game and decision theoretical concepts.

A decision problem consists of a set of alternatives and a complete (but not necessarily transitive) preference relation defined on this set. The class of such decision problems and the class of two-person symmetric games are somewhat tautologically equivalent and can be represented by the same mathematical object. Accordingly, I will consider an identity mapping between those classes. The image of a two-person symmetric game under the mapping is called the equivalent decision problem of the game, and the pre-image of a decision problem is called the equivalent game of the decision problem. 
Chapter 2. Two-person symmetric games and decision problems

In Section 2.2, I will introduce the framework that will be used throughout this chapter. In Section 2.3, I will consider two-person symmetric games in pure strategies and decision problems in a riskless context. I observe that a strategy is an optimal pure threat strategy (Nash, 1953) in a two-person symmetric game if and only if it is a maximal element in its equivalent decision problem. In particular, a pure Nash equilibrium in a two-person symmetric zero-sum game and a pair of maximal elements in its equivalent decision problem coincide.

If a game does not possess a pure Nash equilibrium, a solution in the mixed extension of the game can usually be found. Similarly, if a decision problem does not possess a maximal element, the decision problem can be extended to its "mixed extension" to find a solution. In Section 2.4, I will extend games and decision problems to mixed extension so that a decision maker can choose a probability distribution over the set of alternatives.

Accordingly, I will also show that a two-person symmetric zero-sum game can be extended to its von Neumann-Morgenstern (vN-M) mixed extension if and only if the SSB utility axioms (Fishburn, 1982) continuity, dominance and symmetry are satisfied in its equivalent decision problem. Moreover, I will show that a mixed strategy is optimal (von Neumann, 1928) in a two-person symmetric zero-sum game if and only if it is a maximal element in its equivalent decision problem. More generally, a strategy is an optimal mixed threat strategy in a two-person symmetric game if and only if it is a maximal element in its equivalent decision problem.

In Section 2.5, I will demonstrate that a two-person symmetric game is a potential game if and only if its equivalent decision problem satisfies vN-M utility. ${ }^{1}$ Accordingly, I will provide a formula for the number

\footnotetext{
${ }^{1}$ Roth (1977) relates vN-M utility with another concept: He shows that the Shapley value of a player and vN-M utility coincide if the player's preferences over player positions in the game can be represented by that utility function, and those preferences satisfy some assumptions.
} 
of linearly independent equations for the independence axiom to be satisfied. In effect, the value grows quadratically as the number of alternatives increase, which may be interpreted as an alternative way to show the strength of the independence axiom. Finally, I will present how a puzzling phenomenon in SSB utility (Fishburn, 1984) turns out to be a well-known property of Nash equilibrium.

\subsection{The framework}

Let $(X, u)$ denote a two-person symmetric game, in which players each have the same set of pure actions $X$, which is not necessarily finite. If Player 1 plays $x \in X$ and Player 2 plays $y \in X$, Player 1 receives $u(x, y)$ and Player 2 receives $u(y, x)$, where $u: X \times X \rightarrow \mathbb{R}$. A two-person symmetric zero-sum game is denoted by $(X, v)$ where for all $x$ and $y$, $v(x, y)+v(y, x)=0$.

Let $(X, \succeq)$ denote a decision problem, in which $X$ is a set of alternatives available to a decision maker, and the relation $\succeq \subseteq X \times X$ represents the preferences of the decision maker over $X$, which can be read as weakly preferred or at least as good as. Denote $x \sim y$ if $x \succeq y$ and $y \succeq x$, and $x \succ y$ if $x \succeq y$ but not $x \sim y$.

If we assume that a preference relation on a countable set is complete and transitive, then it is representable by a one-variable order-preserving utility function. ${ }^{2}$ However, this is not possible if we remove the transitivity assumption. In this case, a simple approach to obtain a representation is to use a two-variable utility function. ${ }^{3}$ The following observation states that such a decision problem is representable.

\footnotetext{
${ }^{2}$ See, for example, Debreu (1954).

${ }^{3}$ For example, Fishburn (1982) generalized the expected utility of von Neumann and Morgenstern by a skew-symmetric two-variable utility function without using the transitivity and the independence axioms.
} 
Chapter 2. Two-person symmetric games and decision problems

Observation 1. Suppose that the relation $\succeq$ on a set $X$ is complete. Then, there exists $U: X \times X \rightarrow \mathbb{R}$ such that for all $x$ and $y$ in $X, x \succ y \Leftrightarrow U(x, y)>$ $U(y, x)$.

Proof. Let $\succeq$ be a complete relation on $X$. Define a real valued function $U$ such that for all $(x, y) \in X \times X$ with $x \succ y$, we have $U(x, y)>U(y, x)$. And for all $x \sim y$ define $U(x, y)=U(y, x)$. It is left to show that for $z$ and $w$ in $X, U(z, w)>U(w, z)$ implies that $z \succ w$. Assume that $z \preceq w$. Then, $U(z, w) \leq U(w, z)$, which is a contradiction to our supposition.

Remark 1. Let $U: X \times X \rightarrow \mathbb{R}$ be a function. The preference relation on $X$ defined by $x \succ y$ if $U(x, y)>U(y, x)$ and $x \sim y$ if $U(x, y)=U(y, x)$ would be complete and such $U$ would represent $\succeq$.

The function $U(x, y)$ may be interpreted as a magnitude of the intensity of preference for $x$ over $y$. If the intensity of preference for $x$ over $y$ is greater than the one for $y$ over $x$, then $x$ is preferred to $y$. In particular, for $x$ and $y$ in $X$ the difference $U(x, y)-U(y, x)$ defines another function, say $V(x, y)$, which has a nice property of being skew-symmetric, that is $V(x, y)=-V(y, x)$. The following corollary illustrates this situation.

Corollary 1. If the relation $\succeq$ on a set $X$ is complete, then there exist $V$ : $X \times X \rightarrow \mathbb{R}$ such that for all $x$ and $y$ in $X, x \succ y \Leftrightarrow V(x, y)>0$ with $V(x, y)+V(y, x)=0$.

The triplets $(X, U, \succeq)$ and $(X, V, \succeq)$ are also decision problems. Notice that a function $U$ as in Remark 1 induces a unique preference relation on $X$, hence $(X, U, \succeq)$ is a more specific decision problem than $(X, \succeq){ }^{4}$

Note that given a decision problem $(X, \succeq)$ there are (uncountably) many functions $U$ that represent $\succeq$. We define the set

$$
[\succeq]=\{U: X \times X \rightarrow \mathbb{R} \mid U \text { represents } \succeq\},
$$

\footnotetext{
${ }^{4}$ I will write $(X, U)$ instead of $(X, U, \succeq)$ and each time I refer to a decision problem I will state which form I consider.
} 
2.3. Games in pure strategies and decision problems in a riskless context

which will be helpful to identify the similarities of the functions representing the same relation.

\subsection{Games in pure strategies and decision problems in a riskless context}

\subsubsection{The equivalence mapping}

Let $\mathcal{D}$ denote the class of all decision problems of the form $(X, U)$, where $U$ is an order-preserving utility function as in Observation 1 , and $\mathcal{G}$ denote the class of all two-person symmetric games. The next proposition shows the equivalence between those two classes.

Proposition 1. $\mathcal{G}=\mathcal{D}$.

Proof. To show $\mathcal{G} \subseteq \mathcal{D}$, let $(X, u)$ be a game in $\mathcal{G}$. By Remark 1 , the preference relation defined by $x \succ y$ if $u(x, y)>u(y, x)$ is complete, and $u$ represents $\succeq$. Accordingly, $(X, u, \succeq)$ belongs to the desired class when we consider $X$ as the set of alternatives. Conversely, given a decision problem $(X, U)$, define the two-person symmetric game as follows. Consider $X$ as the set of pure strategies for each player, and when Player 1 plays $x \in X$ and Player 2 plays $y \in X$, Player 1 receives a payoff of $U(x, y)$ and Player 2 receives $U(y, x)$. Consequently, the game $(X, U)$ is a two-person symmetric game.

In particular, the class of decision problems with a representation function $V$ (as in Corollary 1 ) is equivalent to the class of two-person symmetric zero-sum games. Since the construction is essentially the same as the preceding proposition, I have omitted it.

So far, I have considered the pair $(X, u)$ both as a game and as a decision problem. To stress this, I define an identity mapping $\mathcal{T}: \mathcal{G} \rightarrow \mathcal{D}$, which does not change the mathematical object but interprets it differently. In other words, the image of a two-person symmetric game, $(X, u)$, 


\begin{tabular}{c|c|c|c|}
\multicolumn{1}{c}{} & \multicolumn{1}{c}{$\mathrm{x}$} & \multicolumn{1}{c}{$\mathrm{z}$} \\
\cline { 2 - 4 } $\mathrm{x}$ & 1,1 & 3,0 & 0,3 \\
\cline { 2 - 4 } $\mathrm{y}$ & 0,3 & 1,1 & 3,0 \\
\cline { 2 - 4 } $\mathrm{z}$ & 3,0 & 0,3 & 1,1 \\
\cline { 2 - 4 } & & &
\end{tabular}

\begin{tabular}{c|c|c|c|}
\multicolumn{1}{c}{} & \multicolumn{1}{c}{$\mathrm{x}$} & \multicolumn{1}{c}{$\mathrm{y}$} & $\mathrm{z}$ \\
\cline { 2 - 4 } $\mathrm{x}$ & 0,0 & $1,-1$ & $-1,1$ \\
\cline { 2 - 4 } $\mathrm{y}$ & $-1,1$ & 0,0 & $1,-1$ \\
\cline { 2 - 4 } $\mathrm{z}$ & $1,-1$ & $-1,1$ & 0,0 \\
\cline { 2 - 4 } & & &
\end{tabular}

Figure 2.1: Games $(X, u)$ and $(X, v)$ respectively

under the mapping $\mathcal{T}$ gives the decision problem, $(X, u, \succeq)$, where $u$ is the order-preserving utility function representing the relation $\succeq$.

In addition, if $u$ represents the preference relation $\succeq$ on $X$, I call the pair, $(X, \succeq)$, the equivalent decision problem of the two-person symmetric game $(X, u)$. To avoid confusion and to distinguish between decision problems and games, I write $\mathcal{T}((X, u))=(X, U)$ where $u=U$. Then I say the equivalent decision problem of the game $(X, u)$ is $(X, U) .^{5}$

Next, I provide an example to clarify how decision problems and games are related. Figure 2.1 presents a two-person symmetric game, $(X, u)$, and a two-person symmetric zero-sum game $(X, v)$. Consider the equivalent decision problem $(X, U)$ of the game $(X, u)$ under the mapping $\mathcal{T}$ where $U=u$. The preference relation represented by the utility function $U$ is $x \succ y \succ z \succ x$, because $U(x, y)>U(y, x), U(y, z)>$ $U(z, y)$ and $U(z, x)>U(x, z)$. Similarly, consider the equivalent decision problem $(X, V)$ of the game $(X, v)$ under the mapping $\mathcal{T}$, where $V=v$. Since $V(x, y)>0, V(y, z)>0$ and $V(z, x)>0$, the preference relation turns out to be the same as before: $x \succ y \succ z \succ x$. Thus, both games have the same decision problem, $(X, \succeq)$, and both $u$ and $v$ represent $\succeq$, that is, $u$ and $v$ are in $[\succeq]$. In the following section, I will show that different games whose equivalent decision problems have the same preference relation share some common properties.

Notice that the game $(X, v)$ in Figure 1 is the classical Rock-Paper-

\footnotetext{
${ }^{5}$ Those notations will be helpful since I will need to make distinctions between the decision problems of the form $(X, U),(X, V)$ and $(X, \succeq)$.
} 
2.3. Games in pure strategies and decision problems in a riskless context

Scissors game, which is known by its cyclic structure. In other words, the relation defined by "one shape beats the other" is cyclic, because rock beats scissors, scissors beats paper and paper beats rock. Intuitively speaking, it would be expected that the equivalent decision problem (if one could exist) of Rock-Paper-Scissors game should have cyclic preferences, which is actually the case as I showed.

\subsubsection{Counterparts of maximal elements}

$X$ is said to admit a maximal element, $x$, with respect to a preference relation $\succeq$ on $X$ if there exists no element $y \in X$ such that $y \succ x$. If $\succeq$ is complete, this definition reduces to the following: for all $y$ in $X, x \succeq y$.

The following observation illustrates the counterparts of pure Nash equilibria in two-person symmetric zero-sum games.

Observation 2. Let $(X, v)$ be a two-person symmetric zero-sum game and $(X, \succeq)$ be its equivalent decision problem. $X$ admits a maximal element $x^{*}$ with respect to $\succeq$ if and only if the game $(X, v)$ possesses a pure Nash equilibrium $\left(x^{*}, x^{*}\right)$.

Proof. ' $\Rightarrow$ ' Since $(X, \succeq)$ is the equivalent decision problem of the game $(X, v)$, the function $v$ represents the relation $\succeq$ in the sense of Corollary 1 . Hence $x^{*} \succeq y$ if and only if $v\left(x^{*}, y\right) \geq 0$ for all $y \in X$. If $(X, v)$ has a value, it should be 0 because it is a two-person symmetric zero-sum game. The strategy $x^{*}$ guarantees the value 0 , so it is an optimal strategy. By symmetry, the pair $\left(x^{*}, x^{*}\right)$ is a pure Nash equilibrium of $(X, v)$.

' $\Leftarrow$ ' If $\left(x^{*}, x^{*}\right)$ is an equilibrium of the game $(X, v)$, then the value of the game exists and it is 0 . Moreover, $x^{*}$ is an optimal strategy and it guarantees the value 0 , that is, $v\left(x^{*}, y\right) \geq 0$ for all $y \in X$. It implies that $x^{*} \succeq y$ for all $y \in X$, since $v$ represents the relation $\succeq$. Thus, $x^{*}$ is a maximal element.

In two-person symmetric games, it turns out that a maximal element 
Chapter 2. Two-person symmetric games and decision problems

coincides with an optimal threat strategy, which we know from the bargaining problem (Nash, 1953). A strategy is called an optimal threat strategy in a two-person game if it is an optimal strategy of the game that has been obtained from the payoff differences of the original game. ${ }^{6}$

An evolutionary stable strategy for finite populations (Schaffer, 1988) also coincides with an optimal pure threat strategy in two-person symmetric games if the population consists of two individuals and the contest size is also two.

The following observation illustrates the relationship between an optimal pure threat strategy and a maximal element of a decision problem.

Observation 3. Let $(X, u)$ be a two-person symmetric game and $(X, \succeq)$ be its equivalent decision problem. An element $x^{*} \in X$ is maximal with respect to $\succeq$ if and only if $x^{*}$ is an optimal pure threat strategy in $(X, u)$.

Proof. Since $(X, \succeq)$ is the equivalent decision problem of $(X, u)$, the function $u$ represents the relation $\succeq$. Therefore, $x^{*}$ is maximal with respect to $\succeq$ if and only if $u\left(x^{*}, y\right) \geq u\left(y, x^{*}\right)$ for all $y \in X$. This is equivalent to $u\left(x^{*}, y\right)-u\left(y, x^{*}\right) \geq 0$ for all $y \in X$, which implies $x^{*}$ is an optimal strategy in the game derived from the payoff differences of the game $(X, u)$. That is, $x^{*}$ is an optimal threat strategy in $(X, u)$.

To elaborate on Observation 2 and Observation 3, consider a decision problem $(X, \succeq)$. If $X$ admits a maximal element with respect to $\succeq$, all two-person symmetric games $(X, u)$ with $u \in[\succeq]$ have an optimal pure threat strategy, and all two-person symmetric zero-sum games $(X, v)$ with $v \in[\succeq]$ have a pure Nash equilibrium (the reverse implications are also true). The reason is that both $u$ and $v$ represent the same preference relation $\succeq$.

${ }^{6}$ Note that here I use the definition of optimal threat strategy for transferable utility games. See, for example, Owen (1968). 
2.4. Mixed extension of games and decision problems

\subsection{Mixed extension of games and decision prob- lems}

If a game has no Nash equilibrium in pure strategies, the mixed extension of the game is usually considered; that is, players are allowed to randomize over the pure strategies. In case of finite games, the problem of existence of a Nash equilibrium is solved in this way. Similarly, we can think of randomization in decision problems in case there is no maximal element.

Let $(X=\{x, y, z\}, \succeq)$ be a decision problem such that $x \succ y \succ z \succ$ $x$. According to Tullock (1964), a paradoxical situation arises when a decision maker is to choose an alternative from $X$. One way to solve this problem is to allow the decision maker to randomize over the set of alternatives. ${ }^{7}$ Recall the Rock-Paper-Scissors game in Figure 2.1, where the unique Nash equilibrium is the profile in which each player mixes uniformly over the actions. As I showed, the equivalent decision problem of this game is ( $X=\{x, y, z\}, \succeq)$ where $\succeq$ is cyclic. Thus, one may suspect that the solution to the decision problem $(X=\{x, y, z\}, \succeq)$ is also to choose each alternative with equal probability.

\subsubsection{Two-player symmetric zero-sum games}

The mixed extension of a finite two-person symmetric zero-sum game $(X, v)$ is the game $\left(\Delta(X), v^{\prime}\right)$ in which $\Delta(X)$ denotes the set of all probability distributions over $X$ and the von Neumann-Morgenstern utility function $v^{\prime}$ denotes the bilinear extension of $v$ to $\Delta(X) \times \Delta(X)$.

Consider a finite decision problem $(X, V)$ and extend the function $V$ on $X \times X$ to a function $V^{\prime}$ on $\Delta(X) \times \Delta(X)$ such that $V_{\mid X \times X}^{\prime}=V .^{8}$ Accordingly, the preference relation $\succeq$ on $X$ is also extended to $\succeq^{\prime}$ on

\footnotetext{
${ }^{7}$ See, for example, Fishburn (1988, p. 137).

${ }^{8}$ The notation $V_{\mid X \times X}^{\prime}=V$ reads as the restriction of $V^{\prime}$ to the domain $X \times X$.
} 
Chapter 2. Two-person symmetric games and decision problems

$\Delta(X)$ so that $\succeq_{\mid X \times X}^{\prime}=\succeq$. I denote the mixed extension of a decision problem as $\left(\Delta(X), V^{\prime}, \succeq^{\prime}\right)$ or shortly $\left(\Delta(X), V^{\prime}\right)$.

It turns out that there is a connection between the extended preference relation and the SSB (skew-symmetric and bilinear) utility of Fishburn (1982), which is based on the following axioms. For all $p, q$ and $r$ in $\Delta(X)$ :

Axiom C (Continuity). There exists $\beta \in(0,1)$ such that $p \succ q \succ r$ implies $q \sim \beta p+(1-\beta) r$.

Axiom D (Dominance). For all $\alpha \in(0,1), p \succ q$ and $p \succeq r$ implies $p \succ \alpha q+(1-\alpha) r, q \succ p$ and $r \succeq p$ implies $\alpha q+(1-\alpha) r \succ p$, and $p \sim q$ and $p \sim r$ implies $p \sim \alpha q+(1-\alpha) r$.

Axiom S (Symmetry). For all $\alpha \in(0,1), p \succ q \succ r, p \succ r$ and $q \sim \frac{1}{2} p+\frac{1}{2} r$ implies $\alpha p+(1-\alpha) r \sim \frac{1}{2} p+\frac{1}{2} q \Leftrightarrow \alpha r+(1-\alpha) p \sim \frac{1}{2} r+\frac{1}{2} q$.

As the following theorem states, these axioms are necessary and sufficient for representing the preferences by an SSB utility function.

Theorem (Fishburn, 1982). The relation $\succeq$ on $\Delta(X)$ satisfies $C, D$ and $S$ if and only if there exists an SSB function $\phi$ such that for all $p$ and $q$ in $\Delta(X) p \succ q \Leftrightarrow \phi(p, q)>0$. Moreover, $\phi$ is unique up to multiplication by a positive constant.

Let $\mathcal{G}^{\prime} \subset \mathcal{G}$ be the class of two-person symmetric zero-sum games in vN-M mixed extension $\left(\Delta(X), v^{\prime}\right)$, and $\mathcal{D}^{\prime} \subset \mathcal{D}$ be the class of decision problems of the form $\left(\Delta(X), V^{\prime}\right)$. Consider again the identity mapping $\mathcal{T}$ whose domain and codomain are restricted to $\mathcal{G}^{\prime}$ and $\mathcal{D}^{\prime}$, respectively. Note that mapping $\mathcal{T}$ is one-to-one but not onto, because $\mathcal{G}^{\prime} \subset \mathcal{D}^{\prime}$. For instance, there could be decision problems in $\mathcal{D}^{\prime}$ whose representation function does not satisfy bilinearity. To obtain a bijective mapping, we need to impose more assumptions on the preferences of decision problems in $\mathcal{D}^{\prime}$.

Similar to before, I say that a decision problem $\left(\Delta(X), \succeq^{\prime}\right)$ is the equivalent decision problem of a two-person symmetric zero-sum game 
2.4. Mixed extension of games and decision problems

$\left(\Delta(X), v^{\prime}\right)$ if the utility function $v^{\prime}$ under mapping $\mathcal{T}$ represents the relation $\succeq^{\prime}$.

The following proposition shows the relation between mixed extension of a game and its equivalent decision problem.

Proposition 2. Let $(X, v)$ be a two-person symmetric zero-sum game, and $(X, \succeq)$ be its equivalent decision problem. $(X, v)$ can be extended to $v N-M$ mixed extension $\left(\Delta(X), v^{\prime}\right)$ if and only if the preference relation, $\succeq^{\prime}$, of the extended decision problem $\left(\Delta(X), \succeq^{\prime}\right)$ satisfies axioms $C, D$ and $S$.

Proof. ' $\Rightarrow$ ' Let $\left(\Delta(X), v^{\prime}\right)$ be the equivalent decision problem of game $\left(\Delta(X), v^{\prime}\right)$. We show that $v^{\prime}$ is skew-symmetric and bilinear and hence it satisfies axioms $C, D$ and $S$ by the theorem of Fishburn (1982). The function $v^{\prime}$ is skew-symmetric, that is, $v^{\prime}(p, q)=-v^{\prime}(q, p)$ for all $p, q \in$ $\Delta(X)$, because $v^{\prime}(p, q)=p^{\top} A q$, in which $A$ is the payoff matrix of Player 1 that satisfies the condition $A=-A^{\top} .{ }^{9}$ Furthermore, the payoff function $v^{\prime}$ is bilinear by definition.

' $\Leftarrow$ ' We extend the decision problem $(X, \succeq)$ to $\left(\Delta(X), \succeq^{\prime}\right)$. If the relation $\succeq^{\prime}$ satisfies axioms $C, D$ and $S$, then there exist a skew-symmetric bilinear function $\phi$ which is unique up to a multiplication by a positive constant such that $p \succ q$ if and only if $\phi(p, q)>0$, where $\phi(p, q)=$ $\sum_{x \in X} \sum_{y \in X} p(x) q(y) \phi(x, y)$ for all $p, q$ in $\Delta(X)$ (Fishburn, 1982). Since $\phi$ is the extension of $v$, (i.e., $\phi(x, y)=v(x, y)$ for all $x$ and $y$ ), we obtain a unique $\phi$ on $\Delta(X)$, which represents $\succeq^{\prime}$. Define the payoff function $v^{\prime}$ of Player 1 as $v^{\prime}(p, q)=\phi(p, q)$ for all $p, q$ in $\Delta(X)$. Consequently, we obtain the mixed extension, $\left(\Delta(X), v^{\prime}\right)$, of the two-person symmetric zero-sum game $(X, v)$.

Figure 2 illustrates Proposition 2 and its proof. It shows the steps to reach the extended preference relation $\succeq^{\prime}$ on $\Delta(X)$, which satisfies

${ }^{9}$ Note that $p^{\top} A q=p^{\top}\left(-A^{\top}\right) q=\left(p^{\top}\left(-A^{\top}\right) q\right)^{\top}=-q^{\top} A p=-v^{\prime}(q, p)$. 

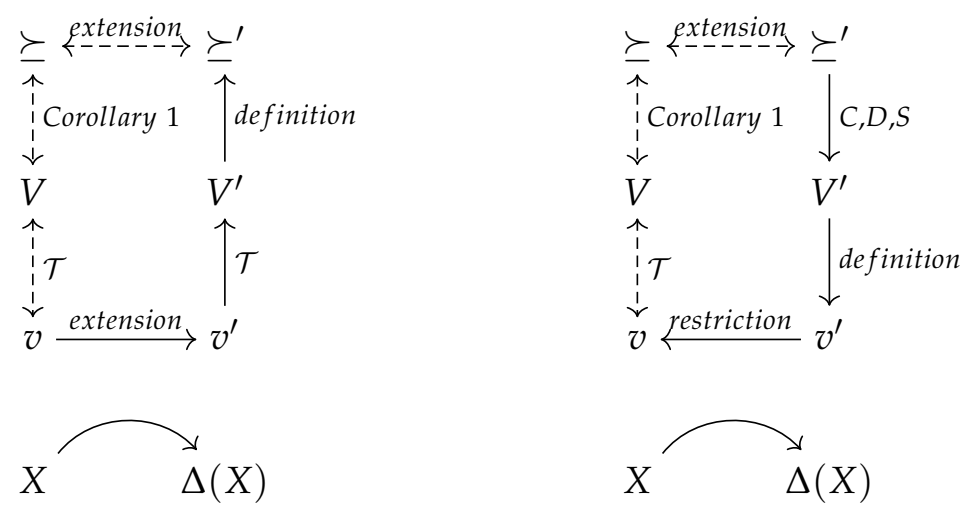

Figure 2.2: Illustration of Proposition 2

axioms $C, D$ and $S$, and vice versa. ${ }^{10}$

Let $\mathcal{D}^{S S B} \subset \mathcal{D}^{\prime}$ be the class of decision problems of the form $(\Delta(X), v)$, where $v$ is an SSB function. The following corollary shows that the identity mapping $\mathcal{T}$ becomes bijective, if we restrict the domain to $\mathcal{G}^{\prime}$ and the codomain to $\mathcal{D}^{S S B}$.

Corollary 2. $\mathcal{G}^{\prime}=\mathcal{D}^{S S B}$.

Regarding the two-person symmetric zero-sum games, the above corollary shows that a similar result to Proposition 1 in mixed strategies can also be obtained. The following observation is an analogue of Observation 2.

Observation 4. Let $(\Delta(X), \succeq)$ be an equivalent decision problem of the twoperson symmetric zero-sum game, $(\Delta(X), v)$. A strategy $p$ is optimal if and only if $p$ is a maximal element with respect to $\succeq$.

\footnotetext{
${ }^{10}$ As an example, start by moving counter-clockwise (solid arrow) from $v$, which is located on the left bottom corner. We can obtain the extended function $v^{\prime}$ from the mixed extension of the game $(X, v)$. Then, we can obtain the extended decision problem $\left(\Delta(X), V^{\prime}\right)$ by applying the mapping $\mathcal{T}$. Accordingly, we define the extended preference relation $\succeq^{\prime}$ induced by $V^{\prime}$, which satisfies the SSB utility axioms $C, D$ and $S$ as shown in Proposition 2. Finally, by taking the restriction of $V^{\prime}$ on $X$ we obtain the decision problem $(X, V)$, hence the non-extended game $(X, v)$.
} 
Proof. ' $\Rightarrow$ ' If $p$ is an optimal strategy, then $v(p, q) \geq 0$ for all $q \in \Delta(X)$. And we have $p \succeq q$ for all $q \in \Delta(X)$, because $v$ represents the relation $\succeq$. ' $\Leftarrow$ ' If $p$ is maximal, then $v(p, q) \geq 0$ for all $q \in \Delta(X)$. Since $p$ guarantees the value of the game, it is an optimal strategy in $(\Delta(X), v)$.

\subsubsection{Two-player symmetric games}

Let $\left(\Delta(X), u^{\prime}\right)$ be the mixed extension of the finite two-person symmetric game $(X, u)$, in which the $\mathrm{vN}-\mathrm{M}$ utility function $u^{\prime}$ is the bilinear extension of $u$ to $\Delta(X) \times \Delta(X)$.

Given a decision problem $(X, U)$, where $X$ is finite, I extend the function $U$ to a function $U^{\prime}$ on $\Delta(X) \times \Delta(X)$ such that $U_{\mid X \times X}^{\prime}=U$. As a result, relation $\succeq$ on $X$ is also extended to $\succeq_{\mid X \times X}^{\prime}$ on $\Delta(X)$.

Let $\mathcal{G}^{\prime \prime} \subset \mathcal{G}$ denote the class of two-person symmetric games in vNM mixed extension $(\Delta(X), u)$, and $\mathcal{D}^{\prime \prime} \subset \mathcal{D}$ denote the class of decision problems of the form $\left(\Delta(X), U^{\prime}\right)$. The following observation illustrates that the vN-M extension of a symmetric game implies SSB utility in its equivalent decision problem.

Observation 5. Let $(X, u)$ be a two-person symmetric game and $(X, \succeq)$ be its equivalent decision problem. If one extends $(X, u)$ to $v N-M$ mixed extension $\left(\Delta(X), u^{\prime}\right)$, then the extended preference relation $\succeq^{\prime}$ on $\Delta(X)$ satisfies axioms $C, D$ and $S$.

Proof. Define $v(p, q)=u(p, q)-u(q, p)$ for all $p$ and $q$ in $\Delta(X)$ so that $p \succeq^{\prime} q \Leftrightarrow v(p, q) \geq 0$. By definition, $v$ is a skew-symmetric function and the bilinearity of $v$ follows from the bilinearity of $u$.

Regarding the other direction, if an extended decision problem satisfies SSB utility, then it is possible to construct a two-person symmetric game which is unique up to payoff differences. That is, $u$ and $u^{\prime}$ are such functions if and only if $u(p, q)-u(q, p)=u^{\prime}(p, q)-u^{\prime}(q, p)$ for 
Chapter 2. Two-person symmetric games and decision problems

all $p$ and $q$ in $\Delta(X)$. Accordingly, $u$ becomes $\Delta$-bilinear-the function $u(p, q)-u(q, p)$ is bilinear for all $p$ and $q$ in the domain of $u$.

Note that the identity mapping $\mathcal{T}$ restricted to domain $\mathcal{G}^{\prime \prime}$ and to codomain $\mathcal{D}^{\prime \prime}$ is one-to-one but not onto. However, it remains unknown which axioms are exactly needed on the extended preferences to have that mapping onto. ${ }^{11}$ The following proposition shows these arguments more formally.

Proposition 3. Let $(X, u)$ be a two-person symmetric game, $(X, u, \succeq)$ its equivalent decision problem, and $\left(\Delta(X), u^{\prime}\right)$ the $v N-M$ mixed extension of the game. If the relation $\succeq^{\prime}$ of the extended decision problem $\left(\Delta(X), U^{\prime}, \succeq^{\prime}\right)$ satisfies axioms $C, D$ and $S$, then $u^{\prime} \in\left[\succeq^{\prime}\right]$. Moreover, any $U^{\prime}$ in $\left[\succeq^{\prime}\right]$ is $\Delta$-bilinear and unique up to payoff differences.

Proof. First, we show that $u^{\prime} \in\left[\succeq^{\prime}\right]$. Define the function $v^{\prime}$ by $v^{\prime}(p, q)=$ $U^{\prime}(p, q)-U^{\prime}(q, p)$ for all $p$ and $q$ in $\Delta(X)$ with $U^{\prime} \in\left[\succeq^{\prime}\right]$, so we have $p \succeq^{\prime} q \Leftrightarrow v^{\prime}(p, q) \geq 0$. If $\succeq^{\prime}$ satisfies axioms $C, D$ and $S$, then $v^{\prime}$ becomes an SSB function by Theorem 1 of Fishburn (1982). Since $U^{\prime}$ and $u^{\prime}$ are the extensions of $u$, we have $v^{\prime}(x, y)=u(x, y)-u(y, x)=$ $u^{\prime}(x, y)-u^{\prime}(y, x)$ for all $x$ and $y$ in $X$. Since both $v^{\prime}$ and $u^{\prime}$ are bilinear, we have $v^{\prime}(p, q)=u^{\prime}(p, q)-u^{\prime}(q, p)$ for all $p$ and $q$ in $\Delta(X)$. Consequently, we obtain $p \succeq^{\prime} q \Leftrightarrow u^{\prime}(p, q) \geq u^{\prime}(q, p)$, that is, $u^{\prime} \in\left[\succeq^{\prime}\right]$.

Second, we show that any function $U^{\prime}$ in $\left[\succeq^{\prime}\right]$ is unique up to the difference in payoffs. Observe that $v^{\prime}$ is uniquely defined on the whole domain since the values of $v^{\prime}(x, y)=u(x, y)-u(y, x)$ are fixed for all $x$ and $y$ in $X$. If $u_{1}$ and $u_{2}$ are in $\left[\succeq^{\prime}\right]$, then we obtain $u_{1}(p, q)-u_{1}(q, p)=$ $u_{2}(p, q)-u_{2}(q, p)$ for all $p$ and $q$ in $\Delta(X)$.

Finally, we show that any $U^{\prime}$ in $\left[\succeq^{\prime}\right]$ is $\Delta$-bilinear. We have

$$
v^{\prime}(\alpha p+(1-\alpha) r, q)=u_{1}(\alpha p+(1-\alpha) r, q)-u_{1}(q, \alpha p+(1-\alpha) r) .
$$

\footnotetext{
${ }^{11}$ It is not obvious whether it is even possible to have a set of axioms on a preference relation such that the representation function $u$ becomes unique (up to a positive linear transformation).
} 
2.5. The independence axiom and potential games

Then, bilinearity of $v^{\prime}$ implies that

$$
\begin{gathered}
\alpha v^{\prime}(p, q)+(1-\alpha) v^{\prime}(r, q) \\
=\alpha u_{1}(p, q)+(1-\alpha) u_{1}(r, q)-\alpha u_{1}(q, p)-(1-\alpha) u_{1}(q, r) .
\end{gathered}
$$

The $\Delta$-bilinearity in the second argument can be shown analogously.

The following observation illustrates a result in mixed strategies that is analogous to Observation 3.

Observation 6. Let $(\Delta(X), u)$ and $\left(\Delta(X), \succeq^{\prime}\right)$ be a two-person symmetric game and its equivalent decision problem, respectively. A strategy $p^{*}$ is maximal with respect to $\succeq^{\prime}$ if and only if $p^{*}$ is an optimal threat strategy.

Proof. Since $u$ represents $\succeq^{\prime}, p^{*}$ is a maximal element if and only if $u\left(p^{*}, q\right) \geq u\left(q, p^{*}\right)$. Thus, $p^{*}$ is an optimal threat strategy in $(\Delta(X), u)$.

Note that for any $u^{\prime} \in\left[\succeq^{\prime}\right]$, the game $\left(\Delta(X), u^{\prime}\right)$ is a two-person symmetric game, but it is not necessarily the vN-M mixed extension of the game $\left(X, u^{\prime}\right)$. However, all the games $\left(\Delta(X), u^{\prime}\right)$ with $u^{\prime} \in\left[\succeq^{\prime}\right]$ have the same optimal threat strategy $p^{*}$, because the payoff functions represent the same preference relation.

\subsection{The independence axiom and potential games}

A difference between expected utility and SSB utility is that transitivity and the axiom of independence is not assumed in SSB utility. If a decision problem satisfies the independence axiom, I observe that its equivalent two-person symmetric game becomes a potential game and the game matrix satisfies a "triangular equality" property. The following definitions and proposition show this more formally. 
It appears that the subclass of potential games were proposed by Rosenthal (1973) and later formalized by Monderer and Shapley (1996). It is a useful class because all potential games possess a pure Nash equilibrium. Moreover, the potential function can be used for Nash equilibrium selection. ${ }^{12}$ The following defines potential games in twoperson symmetric games.

Definition 1. A symmetric two-person game $(X, u)$ is called a (exact) potential game if there exists a function $P: X \times X \rightarrow \mathbb{R}$ such that for all $y$ and all $x, z$ in $X$, we have $u(x, y)-u(z, y)=P(x, y)-P(z, y)$ and $u(x, y)-u(z, y)=P(y, x)-P(y, z)$.

A preference relation $\succeq$ on $\Delta(X)$ satisfies the independence axiom ${ }^{13}$ if for all $p, q$ and $r$ in $\Delta(X)$ and for all $\alpha \in(0,1)$ such that $p \succeq q \Leftrightarrow$ $\alpha p+(1-\alpha) r \succeq \alpha q+(1-\alpha) r$.

If $u$ represents the relation $\succeq$ on $\Delta(X)$, then we can express the independence in terms of $u$ as follows. For every $p, q$ and $r$ in $\Delta(X)$ and for all $\alpha \in(0,1)$, we have

$$
\begin{gathered}
u(p, q) \geq u(q, p) \Leftrightarrow \\
u(\alpha p+(1-\alpha) r, \alpha q+(1-\alpha) r) \geq u(\alpha q+(1-\alpha) r, \alpha p+(1-\alpha) r) .
\end{gathered}
$$

In addition, if the relation $\succeq$ satisfies SSB utility, then $u$ is $\Delta$-bilinear by Proposition 3 and it implies that

$$
\begin{gathered}
u(p, q) \geq u(q, p) \Leftrightarrow \\
\alpha[u(p, q)-u(q, p)]+(1-\alpha)[u(p, r)-u(r, p)+u(r, q)-u(q, r)] \geq 0 .
\end{gathered}
$$

\footnotetext{
${ }^{12}$ For more details, see Monderer and Shapley (1996).

${ }^{13}$ Axiom of vN-M in the form of Jensen (1967). Actually, for my purpose, it is enough to use a weaker independence axiom with $\alpha=\frac{1}{2}$. However, I used the most known version since SSB utility axioms together with weak independence imply the independence axiom. See Fishburn (1982) for details.
} 
2.5. The independence axiom and potential games

The following lemma will be useful for the proposition that follows. It illustrates that the payoff function of a two-person symmetric game satisfies a "triangular equality" property in pure strategies if and only if it satisfies an analogous property in mixed strategies.

Lemma 1. Let $(\Delta(X), u)$ be a two-person symmetric game. For all $x, y, z$ in $X, u(x, y)+u(y, z)+u(z, x)=u(x, z)+u(z, y)+u(y, x)$ if and only if

$$
u(p, q)+u(q, r)+u(r, p)=u(p, r)+u(r, q)+u(q, p)
$$

for all $p, q, r$ in $\Delta X$.

Proof. ' $\Rightarrow$ ' By bilinearity of $u$, we have $u(p, q)=u_{p, q}=\sum_{x, y \in X} p_{x} q_{y} u_{x, y}$.

Substitute $u_{x, y}$ with $u_{x, z}+u_{z, y}+u_{y, x}-u_{y, z}-u_{z, x}$. Then for all $z \in X$, we have

$$
\begin{aligned}
& u_{p, q}=\sum_{x, y \in X} p_{x} q_{y}\left(u_{x, z}+u_{z, y}+u_{y, x}-u_{y, z}-u_{z, x}\right) \\
& \begin{array}{c}
=\sum_{x, y \in X} p_{x} q_{y} u_{x, z}+\sum_{x, y \in X} p_{x} q_{y} u_{z, y}+\sum_{x, y \in X} p_{x} q_{y} u_{y, x}-\sum_{x, y \in X} p_{x} q_{y} u_{y, z} \\
\quad-\sum_{x, y \in X} p_{x} q_{y} u_{z, x} \\
\stackrel{(1)}{=} \sum_{x \in X} p_{x} u_{x, z} \sum_{y \in X} q_{y}+\sum_{x \in X} p_{x} \sum_{y \in X} q_{y} u_{z, y}+u_{q, p}-\sum_{x \in X} p_{x} \sum_{y \in X} q_{y} u_{y, z} \\
\quad-\sum_{x \in X} p_{x} u_{z, x} \sum_{y \in X} q_{y} \\
\stackrel{(2)}{=} \sum_{x \in X} p_{x} u_{x, z}+\sum_{y \in X} q_{y} u_{z, y}+u_{q, p}-\sum_{y \in X} q_{y} u_{y, z}-\sum_{x \in X} p_{x} u_{z, x} \\
\stackrel{(3)}{=} \sum_{x \in X}\left(p_{x}-q_{x}\right) u_{x, z}+\sum_{x \in X}\left(q_{x}-p_{x}\right) u_{z, x}+u_{q, p} .
\end{array}
\end{aligned}
$$

We obtain (1) by rearranging the sums. Then, we obtain (2) by setting $\sum_{x \in X} p_{x}=1$. Finally, by rearranging the index, we obtain (3). Following the same steps as above yields the following equations:

$$
\begin{aligned}
& u_{q, r} \stackrel{(4)}{=} \sum_{x \in X}\left(q_{x}-r_{x}\right) u_{x, z}+\sum_{x \in X}\left(r_{x}-q_{x}\right) u_{z, x}+u_{r, q}, \\
& u_{r, p} \stackrel{(5)}{=} \sum_{x \in X}\left(r_{x}-p_{x}\right) u_{x, z}+\sum_{x \in X}\left(p_{x}-r_{x}\right) u_{z, x}+u_{r, q} .
\end{aligned}
$$


Chapter 2. Two-person symmetric games and decision problems

Summing (3), (4) and (5) up yields $u_{p, q}+u_{q, r}+u_{r, p}=u_{p, r}+u_{r, q}+$ $u_{q, p}$.

$' \Leftarrow$ ' This holds by definition.

The following remark will be useful in the proof of the next proposition.

Remark 2. Given $(X, v)$, the following equations are equivalent for all $x, y, z$ and $w$ in $X$.

$$
\begin{gathered}
v(x, y)+v(z, w)=v(x, w)+v(z, y) \\
v(x, y)=v(x, z)+v(z, y)
\end{gathered}
$$

Proof. ' $\Rightarrow$ ' If $y=z$, then Equation 2.5.3 reduces to Equation 2.5.4.

$' \Leftarrow$ ' By Equation 2.5.4, $v(x, y)=v(x, z)+v(z, y)$ and $v(z, w)=v(z, x)+$ $v(x, w)$. Summing up those two equations yield Equation 2.5.3.

The following proposition illustrates the relationship between potential games and the independence axiom.

Proposition 4. Let $G=(\Delta(X), u)$ be a two-person symmetric game and $(\Delta(X), \succeq)$ be its equivalent decision problem. The following statements are equivalent.

1. $\succeq$ satisfies the independence axiom.

2. $G$ is a potential game.

3. $u(x, y)+u(y, z)+u(z, x)=u(x, z)+u(z, y)+u(y, x)$ for all $x, y, z$ in $X$.

Proof. (1) $\Rightarrow(3)$ : By Observation 5, the equivalent decision problem of the game $(\Delta(X), u)$ satisfies $C, D$ and $S$. If, in addition, it satisfies the independence, then, by Proposition 1 of Fishburn (1982, p. 37), a real valued function $\bar{u}$ exists such that $u(p, q)-u(q, p)=\bar{u}(p)-\bar{u}(q)$ for all 
2.5. The independence axiom and potential games

$p, q$ in $\Delta(X)$. Then it follows for all $x, y, z$ in $X$ we have $u(x, y)-u(y, x)+$ $u(y, z)-u(z, y)+u(z, x)-u(x, z)=\bar{u}(x)-\bar{u}(y)+\bar{u}(y)-\bar{u}(z)+\bar{u}(z)-$ $\bar{u}(x)=0$.

$(3) \Rightarrow(1)$ : By Lemma 1, condition (3) holds if and only if Equation 2.5.2 holds. Then, substituting $u(p, r)-u(r, p)+u(r, q)-u(q, r)$ with $u(p, q)-u(q, p)$ reduces Equation 2.5.1 to $u(p, q) \geq u(q, p) \Leftrightarrow u(p, q) \geq$ $u(q, p)$. Thus, independence is satisfied.

$(2) \Leftrightarrow(3)$ : We will use a sequence of equivalent statements for the demonstration. Define the two-person symmetric zero-sum game $(X, v)$ such that $v(x, y)=u(x, y)-u(y, x)$ for all $x, y$ and $z$ in $X$. Then, by Theorem 20 of Duersch et al. (2012a), $(X, u)$ is a potential game if and only if $(X, v)$ is a potential game. The latter holds if and only if Equation 2.5.3 holds according to Lemma 2.1 of Potters et al. (1999). Remark 2 concludes the proof since Equation 2.5.4 is equivalent to the statement in (3) by definition of $v$.

Corollary 3. A decision problem satisfies von Neumann-Morgenstern utility if and only if its equivalent symmetric game is a potential game.

Roth (1977) showed that the Shapley value of a player in a game coincides with the vN-M utility function that represents his preferences over the player positions in the game. ${ }^{14}$ Consider a decision problem whose set of alternatives consists of different positions of players in a game. If the decision maker has preferences satisfying the axioms of Roth (1977) over the positions, then the equivalent game of the decision problem is a potential game if and only if the Shapley value (of the decision maker in the initial game) is the vN-M utility function that represents the decision maker's preferences in the extended decision problem.

Among the axioms of expected utility of vN-M, perhaps indepen-

\footnotetext{
${ }^{14}$ The vN-M utility function should be normalized. For details, see Roth (1977).
} 
Chapter 2. Two-person symmetric games and decision problems

dence is the most attacked axiom partly due to its strong implications. For example, the Allais paradox illustrates a systematic violation of this axiom. Below, I analyze the effects of imposing the independence axiom on a decision problem. The next proposition gives a formula to obtain the number of linearly independent equations that must be satisfied for triangular equality property to hold.

Proposition 5. Given a matrix $\left[u_{i j}\right]_{n \times n}$ with $n \geq 3$, let the property $(P)$ be such that for all $i, j$ and $k$ we have $u_{i j}+u_{j k}+u_{k i}=u_{i k}+u_{k j}+u_{j i}$ for $i, j, k=1,2, \ldots, n$. Then the number of linearly independent equations needed for $(P)$ to be satisfied is given by $\frac{(n-1)(n-2)}{2}$.

Proof. Let $E_{i j k}$ denote the equation $u_{i j}+u_{j k}+u_{k i}=u_{i k}+u_{k j}+u_{j i}$, and $E$ denote the set of all such equations. We can restrict the attention to the case $i<j<k$, because the equations $E_{i j k}, E_{i k j}, E_{j i k}, E_{j k i}, E_{k j i}$ and $E_{k i j}$ represent the same equation. Keeping the figure below in mind, the sums do not change no matter which node we start summing and whether we go first clockwise or counter clockwise.

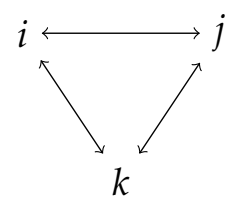

We show that $E^{\prime}=\left\{E_{1 l m} \mid 1<\ell<m\right\}$ is a basis for $E$. To see this, first notice that for any quadruple $i<\ell<k<m$, the equations $E_{i j k}$, $E_{i j m}$ and $E_{i k m}$ imply the equation $E_{j k m}$. Subsequently, it follows that any equation $E_{i j k}$ with $i \neq 1$ can be obtained from equations $E_{1 i j}, E_{1 i k}$ and $E_{1 j k}$. It is left to show that an equation in $E^{\prime}$ cannot be obtained as a linear combination of the other equations in $E^{\prime}$. By contraposition, suppose $E_{1 j k}$ is a linear combination of equations in $E^{\prime} \backslash\left\{E_{1 j k}\right\}$. Then, there exists at least one equation in $E^{\prime}$ which contains the number $u_{j k}$ (otherwise the $u_{j k}$ can never appear). However, this equation must then be $E_{1 j k}$, which 
2.5. The independence axiom and potential games

contradicts our supposition. The number of equations in $E^{\prime}$ is precisely the number given in the proposition.

Proposition 5 gives a formula as a function of strategies for the number of linearly independent equations that must be satisfied for a potential function to exist in two-person symmetric games. This might also indicate the strength of the independence axiom.

Even though it weakens the independence axiom of vN-M utility, SSB utility theory is not problem-free either. For instance, Fishburn (1984, p. 139) mentions some potentially disturbing implications of SSB utility in the case of cycling preferences.

Consider the decision problem $(\Delta(X), v)$ with SSB utility, where $X=\{x, y, z\}$ and $x \succ y \succ z \succ x$. Suppose that we replace $z$ by $z^{\prime}$ such that $z^{\prime}$ in $X^{\prime}=\left\{x, y, z^{\prime}\right\}$ does better than $z$ against $x$ and against $y$, but preferences still cycle, that is, $v\left(z^{\prime}, x\right)>v(z, x)>0$ and $v(y, z)>$ $v\left(y, z^{\prime}\right)>0$. Let $p$ and $p^{\prime}$ be the maximal elements in $(\Delta(X), v)$ and $\left(\Delta\left(X^{\prime}\right), v\right)$, respectively. Then, we might have the following inequality $p\left(z^{\prime}\right)<p(z)$, which seems to be unintuitive because $z^{\prime}$ is better than $z$ with respect to both $x$ and $y$.

However, this situation is a known phenomenon of mixed strategy Nash equilibria in strategic games. Consider the equivalent two-person zero-sum game, $(\Delta(X), v)$, from the above decision problem. All else being equal, if a particular payoff of a player is increased, the action that leads to this payoff sometimes becomes less likely to be played in the Nash equilibrium.

For example, consider the rock-paper-scissors from Figure 2.1. Suppose that the payoff of $v(z, x)$ increased from 1 to 2 and the payoff of $v(z, x)$ from -1 to $\frac{-1}{2}$, and that the payoffs were rearranged so that it remains a symmetric zero-sum game. Then, the probability of $z$ being played at the Nash equilibrium decreases from $\frac{1}{3}$ to $\frac{2}{7}$. 


\subsection{Conclusion}

In this chapter, I have showed that there is an equivalence relation between the class of two-person symmetric games and the class of oneperson decision problems, in which the preferences are complete but not necessarily transitive. By making use of this, I have illustrated some equivalences between the existing notions in games and in decision problems.

For example, I showed that a two-person symmetric zero-sum game can be extended to its von Neumann-Morgenstern mixed extension if and only if the extended decision problem satisfies the SSB utility axioms. In zero-sum symmetric games, I also showed that a strategy is an optimal strategy if and only if it is a maximal element in the equivalent decision problem of the game. Regarding two-person symmetric games, I proved that an optimal threat strategy and a maximal element of its equivalent decision problem coincide.

In addition, I demonstrated that a two-person symmetric game is a potential game if and only if its equivalent decision problem satisfies von Neumann-Morgenstern utility. Accordingly, I provided a formula for the number of linearly independent equations that must be satisfied to pass from SSB utility to von Neumann-Morgenstern utility. This number grows quadratically as the number of alternatives increase. This may be interpreted as an alternative way to show the strength of the independence axiom. 


\section{Chapter 3}

\section{Existence of pure equilibrium in two-person symmetric zero-sum games}

\subsection{Introduction}

In Chapter 2, I showed that there is an equivalence relation between the class of two-person symmetric zero-sum games and the class of decision problems with a complete preference relation. Accordingly, I also showed that a strategy is a pure optimal strategy in a two-person symmetric zero-sum game if and only if it is a maximal element in its equivalent decision problem.

In this chapter, I will exploit this equivalence relation to introduce and analyze three sufficient conditions for the existence of a pure equilibrium in two-person symmetric zero-sum games, and compare them with the extant conditions. The most general condition that I will explore is the socalled sign-quasiconcavity, which generalizes both generalized ordinal potentials (Monderer and Shapley, 1996) and quasiconcavity (Duersch et al., 2012a).

A game is called sign-quasiconcave if it is quasiconcave with respect 
Chapter 3. Existence of pure equilibrium in two-person symmetric zero-sum games

to the sign of the payoff function. I will show that if a two-person symmetric zero-sum game is sign-quasiconcave, it possesses an equilibrium in pure strategies. I will provide an example of a game that is signquasiconcave but neither has it generalized ordinal potential nor it is quasiconcave.

Finally, by several observations, examples and counter-examples, I will compare the generality of the sufficient conditions in two-person symmetric zero-sum games, and illustrate their interrelations in Figure 3.3.

\subsection{The framework}

To recall from Chapter 2, the pair $(X, v)$ denotes a two-person symmetric zero-sum game, where both players have the same set of pure actions $X$, and $v: X \times X \rightarrow \mathbb{R}$ is the utility function of, say, Player 1 . The utility function of Player 2 is the transpose of $v$ satisfying $v(x, y)=-v^{\top}(x, y)$ for all $x$ and $y$ in $X$. The pair $(X, \succeq)$ denotes a decision problem where $X$ is the set of alternatives, and the relation $\succeq \subseteq X \times X$ represents the preferences of the decision maker on $X$, which is assumed to be complete but not necessarily transitive. A decision problem can be interpreted as if a decision maker would choose an element based on her preferences.

Since I do not assume that preferences are transitive, it is not generally possible to represent the preferences by a one-variable order-preserving utility function. In such case, a more convenient approach to obtain a representation is to consider a two-variable utility function. A realvalued function $v$ represents the relation $\succeq$ if for all $x$ and $y$ in $X, v(x, y)>$ 0 if and only if $x \succ y$. In addition, if the function $v$ represents the preference relation $\succeq$ on $X$, I call the pair $(X, \succeq)$ the equivalent decision problem of the two-person symmetric zero-sum game $(X, v)$.

Recall Observation 2 from Chapter 2: $X$ admits a maximal element 
$x^{*}$ with respect to $\succeq$ in $(X, \succeq)$ if and only if its equivalent game $(X, v)$ possesses a pure equilibrium $\left(x^{*}, x^{*}\right)$. This result is useful for deriving sufficient conditions for the existence of a pure equilibrium in two-person symmetric zero-sum games. The idea is to use a basic structure of preference relations, such as transitivity and acyclicity, to obtain a sufficiency result for two-person symmetric zero-sum games.

Definition 2. A preference relation $\succeq$ on a set $X$ is said to be acyclic if for all finite subsets $\left\{x_{1}, x_{2}, \ldots, x_{n}\right\} \subset X$,

$$
x_{1} \succ x_{2}, x_{2} \succ x_{3}, \ldots, x_{n-1} \succ x_{n} \text { implies } x_{1} \succeq x_{n} \text {. }
$$

\subsection{Sufficient conditions}

The following proposition uses a general sufficient condition for the existence of a maximal element to draw a conclusion in two-person symmetric zero-sum games.

Proposition 6. Let $(X, v)$ be a two-person symmetric zero-sum game where $X$ is a compact set in a topological space and $(X, \succeq)$ be its equivalent decision problem. Suppose that the relation $\succeq$ is acyclic such that for all $x \in X$ the set $\{y \in X \mid x \succ y\}$ is open in the relative topology of $X$. Then, the game $(X, v)$ possesses a pure equilibrium.

Proof. If the hypotheses of the proposition are satisfied, and if the preference relation $\succeq$ is acyclic, then $X$ admits a maximal element $x^{*} \in X$ with respect to $\succeq$ by Bergstrom (1975). ${ }^{1}$ Hence, by Observation 2, the game $(X, v)$ possesses a pure equilibrium.

Corollary 4. Let $(X, v)$ be a finite two-person symmetric zero-sum game whose equivalent decision problem is $(X, \succeq)$. If the relation $\succeq$ is acyclic, then $(X, v)$ possesses a pure equilibrium.

\footnotetext{
${ }^{1}$ Although there are more general sufficient conditions on the existence of a maximal element in the literature, I used this one due to its simplicity. Note also that this result has been independently discovered by several authors. See Walker (1977) for a discussion.
} 
Chapter 3. Existence of pure equilibrium in two-person symmetric zero-sum games

Remark. Clearly, if a relation is transitive, then it is acyclic. Thus, assuming transitivity of $\succeq$ instead of acyclicity does not change the conclusion in Corollary 4.

The following definition gives a quasiconcavity-based sufficient condition with respect to the equivalent decision problem of a game.

Definition 3. A symmetric two-person zero-sum game $(X, v)$ is said to be $\succeq$-quasiconcave if for every $x, y$ and $z$ in $X$ with $x \succeq y \succeq z$,

$$
\operatorname{sgn}(v(x, z)) \geq \min \{\operatorname{sgn}(v(x, y)), \operatorname{sgn}(v(y, z))\} .
$$

The following proposition shows that $\succeq$-quasiconcavity is sufficient for the existence of pure equilibrium.

Proposition 7. Suppose that a finite two-person symmetric zero-sum game is $\succeq$-quasiconcave. Then, it possesses a pure equilibrium.

Proof. Let $(X, \succeq)$ be the equivalent decision problem of $(X, v)$. The relation $\succeq$ will be shown as acyclic, so it has a maximal element corresponding to a pure equilibrium of $(X, v)$. To reach a contradiction, suppose that there are $x_{i}^{\prime}$ 's such that $x_{1} \succ x_{2}, x_{2} \succ x_{3}, \ldots, x_{n-1} \succ x_{n}$ and $x_{n} \succ x_{1}$. By $\succeq$-quasiconcavity, $x_{i} \succ x_{j}, x_{j} \succ x_{k}$ implies $x_{i} \succ x_{k}$. Thus, we obtain that $x_{1} \succ x_{n} \succ x_{1}$, which is a contradiction.

The following sufficient condition is a functional representation of Axiom C.3 in Fishburn and Rosenthal (1986). It is an axiom for a preference relation $\succeq^{\prime}$ on a set $X \times X$ to ensure (together with some other axioms) that a specific relation on $X$ is a weak order.

Proposition 8. Let $(X, v)$ be a finite two-person symmetric zero-sum game. If for all $x, y, z$ and $w$ in $X$ the inequality $v(x, y)>v(z, w)$ implies $v(x, z)>$ $v(y, w)$, then $(X, v)$ possesses a pure equilibrium.

Proof. Let $(X, \succeq)$ be the equivalent decision problem of $(X, v)$. First, $\succeq$ will be shown as transitive. If either $x \succ y \succeq z$ or $x \sim y \succ z$, then by 
our supposition $v(x, y)>v(z, y)$ implies $v(x, z)>0$ or $x \succ z$. This also implies that if $x \sim y \sim z$, then neither $x \succ z$ nor $z \succ x$ is possible. Thus, the relation $\succeq$ is transitive, which implies $(X, \succeq)$ has a maximal element corresponding to a pure equilibrium of $(X, v)$.

Quasiconcave games were introduced by Radzik (1991) and may be interpreted as a "discrete version" of quasiconcavity of payoff functions. In two-person symmetric zero-sum games, if each column has a "single peak," the game is called quasiconcave. By exploiting the symmetry feature, Duersch et al. (2012a) showed that if a two-person symmetric zero-sum game is quasiconcave, then it has a pure equilibrium. Moreover, if these games are quasiconcave, then the simple decision rule "imitateif-better" cannot be exploited indefinitely (Duersch et al., 2012b). Next, a notion weaker than quasiconcavity will be described.

Definition 4. A symmetric two-person zero-sum game $(X, v)$ is said to be sign-quasiconcave if there exists a total order $>$ on $X$ such that for every $x, y, z$ and every $w$ in $X$ with $x>y>z$,

$$
\operatorname{sgn}(v(y, w)) \geq \min \{\operatorname{sgn}(v(x, w)), \operatorname{sgn}(v(z, w))\}
$$

To put it differently, the game $(X, v)$ is sign-quasiconcave if and only if the game $(X, \operatorname{sgn}(v))$ is quasiconcave. The following proposition establishes a connection between sign-quasiconcave games and their equivalent decision problems.

Lemma 2. Let $(X, v)$ be a two-person symmetric zero-sum game. If the game is sign-quasiconcave, then the relation $\succeq$ is acyclic in its equivalent decision problem $(X, \succeq)$.

Proof. First, notice that the equivalent decision problem $(X, \succeq)$ of $(X, v)$ and $(X, \operatorname{sgn}(v))$ are the same, because for every $x$ and $y$ in $X, v(x, y)>0$ if and only if $\operatorname{sgn}(v(x, y))>0$. 
Chapter 3. Existence of pure equilibrium in two-person symmetric zero-sum games

We will show that if $\succeq$ on $X$ is cyclic, the game $(X, v)$ is not signquasiconcave. The cyclicity of $\succeq$ implies that there exists a finite subset $\bar{X} \subseteq X$ that does not contain a maximal element. So, by Observation 2, the two-person symmetric zero-sum game $\left(\bar{X}, \operatorname{sgn}\left(v_{\mid \bar{X}}\right)\right)^{2}$ has no pure equilibrium. Based on Theorem 1 in Duersch et al. (2012a), this implies that the game $\left(\bar{X}, \operatorname{sgn}\left(v_{\mid X}\right)\right)$ is not quasiconcave. If it is not possible to find a total order on $\bar{X}$ such that the game $\left(\bar{X}, \operatorname{sgn}\left(v_{\mid \bar{X}}\right)\right)$ is quasiconcave, then it is not possible to find a total order on $X$ so that the larger game $(X, \operatorname{sgn}(v))$ is quasiconcave. Thus, $(X, \operatorname{sgn}(v))$ is not quasiconcave.

Proposition 9. Suppose that a finite two-person symmetric zero-sum game $(X, v)$ is sign-quasiconcave. Then, it possesses a pure equilibrium.

Proof. By Lemma 2, sign-quasiconcavity of $(X, v)$ implies acyclicity of $\succeq$ in its equivalent decision problem. By Corollary $4,(X, v)$ has a pure equilibrium.

\subsection{Relations between sufficient conditions}

The following observation demonstrates that quasiconcavity implies sign-quasiconcavity. However, the reverse implication does not hold as the game in Figure 3.1 exemplifies.

Observation 7. If a two-person symmetric zero-sum game is quasiconcave, then it is sign-quasiconcave.

Proof. By contraposition, assume that $(X, v)$ is not sign-quasiconcave. Then, for every total order $>$ there exists $x, y, z$ and $w$ in $X$ with $x>y>z$ such that $\operatorname{sgn}(v(y, w))<\operatorname{sgn}(v(x, w))$ and $\operatorname{sgn}(v(y, w))<\operatorname{sgn}(v(z, w))$. There are six ways that can occur: (1) $\operatorname{sgn}(v(y, w))$ is either 0 or -1 , $\operatorname{sgn}(v(x, w))$ and $\operatorname{sgn}(v(z, w))$ each are either 0 or 1 . If $\operatorname{sgn}(v(x, w))=1$,

\footnotetext{
${ }^{2}$ The notation $v_{\mid \bar{X}}$ denotes the function $v$ restricted to the domain $\bar{X}$.
} 
$\operatorname{sgn}(v(y, w))=0$ and $\operatorname{sgn}(v(z, w))=1$, then $v(y, w)=0, v(x, w)>0$ and $v(z, w)>0$. Thus, $(X, v)$ is not quasiconcave. The other cases can be shown analogously, so quasiconcavity implies sign-quasiconcavity.

The notion of potential games is introduced by Monderer and Shapley (1996). It is a sufficient condition for the existence of a pure equilibrium in normal-form games. The following defines generalized ordinal potential games in two-person symmetric zero-sum games.

Definition 5. Let $(X, v)$ be a symmetric two-person zero-sum game. If there exists a function $P: X \times X \rightarrow \mathbb{R}$ such that for all $y$ and all $x, z$ in $X$,

$$
\begin{gathered}
v(x, y)-v(z, y)>0 \text { implies } \\
P(x, y)-P(z, y)>0 \text { and } P(y, x)-P(y, z)>0,
\end{gathered}
$$

then $(X, v)$ is called a generalized ordinal potential game. Moreover, if $P$ satisfies

$$
v(x, y)-v(z, y)=P(x, y)-P(z, y)=P(y, x)-P(y, z)
$$

then $(X, v)$ is called an exact potential game.

The following observation illustrates that a generalized ordinal potential game is $\succeq$-quasiconcave, and the equivalent decision problem of such a game is transitive.

Observation 8. Let $(X, v)$ be a two-person symmetric zero-sum game and $(X, \succeq)$ be its equivalent decision problem. If $(X, v)$ has a generalized ordinal potential, then $\succeq$ is transitive and $(X, v)$ is $\succeq$-quasiconcave.

Proof. By contraposition, three cases will show that if $\succeq$ is not transitive, then $(X, v)$ has no generalized ordinal potential.

Case 1: Suppose there exists $x, y$ and $z$ in $X$ with $x \succ y \succ z \succ$ $x$. Then the decision problem $(\{x, y, z\}, \succeq)$ has no maximal element 
Chapter 3. Existence of pure equilibrium in two-person symmetric zero-sum games

which implies the game $\left(\{x, y, z\}, v_{\mid\{x, y, z\}}\right)$ associated to it has no pure equilibrium by Observation 2. Thus, it does not have a generalized ordinal potential by Monderer and Shapley (1996, pp. 128-129). Since $\left(\{x, y, z\}, v_{\mid\{x, y, z\}}\right)$ does not possess a generalized ordinal potential, the game $(X, v)$ also does not possess. ${ }^{3}$

Case 2: Suppose there are $x, y$ and $z$ in $X$ with $x \succ y \succ z \sim x$. It implies $v(x, y)>0, v(y, z)>0$ and $v(x, z)=0$. Accordingly, a potential function must satisfy the following impossible sequence of relations: $P(z, x)>P(y, x)>P(y, z)>P(x, z)>P(x, y)>P(z, y)>P(z, x)$.

Case 3: Suppose there exists $x, y$ and $z$ in $X$ with $x \succ y \sim z \sim x$ which implies $v(x, y)>0, v(y, z)=0$ and $v(x, z)=0$. It further implies that a potential function must satisfy $P(z, x)>P(y, x)>P(y, z)=P(x, z)>$ $P(x, y)>P(z, y)=P(z, x)$, which leads to a contradiction again.

Finally, assume by contradiction that $x \succeq y \succeq z$ exists such that $v(x, z)<\min \{v(x, y), v(y, z)\}$. Then, a potential function for the game $(X, v)$ fails to satisfy the following sequence of relations: $P(z, x)>$ $P(y, x)>P(y, z)>P(x, z)>P(x, y)>P(z, y)>P(z, x)$.

It is an open question whether the conditions given in Observation 8 are sufficient for the existence of a generalized ordinal potential. However, when the game is an exact potential game it is possible to characterize it with respect to its equivalent decision problem. As it might be expected due to a stronger requirement of exact potential games, the equivalent decision problems additionally satisfy the independence axiom. In Chapter 2, I showed that a two-person symmetric (not necessarily zero-sum) game is an exact potential game if and only if its equivalent decision problem satisfies von Neumann-Morgenstern utility. The following observation shows that exact potential games imply that the condition in Proposition 8 is satisfied.

\footnotetext{
${ }^{3}$ Clearly this is true for any game because if it is not possible to construct a generalized ordinal potential for $v_{\mid \bar{X}}$ where $\bar{X} \subseteq X$, then it is not possible to construct one for $v$.
} 


\subsection{Relations between sufficient conditions}

Observation 9. If a two-person symmetric zero-sum game has an exact potential, then the condition in Proposition 8 is satisfied.

Proof. Since $(X, v)$ is an exact potential game we have $v(x, y)=v(x, z)+$ $v(z, y)$ and $v(z, w)=v(z, y)+v(y, w)$. Thus, $v(x, y)>v(z, w)$ implies that $v(x, z)>v(z, w)$.

The following proposition illustrates that transitivity implies signquasi-concavity in finite games.

Proposition 10. Suppose that $\succeq$ is transitive in the equivalent decision problem $(X, \succeq)$ of a finite two-person symmetric zero-sum game $(X, v)$. Then, the game $(X, v)$ is sign-quasiconcave.

Proof. Take an element in $X$ which is minimal with respect to $\succeq$, and call it $x_{1}$. Take a minimal element from $X \backslash\left\{x_{1}\right\}$, and call it $x_{2}$. Then, take a minimal element in $X \backslash\left\{x_{1}, x_{2}\right\}$, and call it $x_{3}$. Continue the process until no element remains in $X$. Define the total order $>$ on $X$ as follows: $x_{j}>x_{i}$ whenever $j>i$. Note also that we have defined $>$ such that $j>i$ only if $x_{j} \succeq x_{i}$. We show that for every $x_{k}, x_{j}, x_{i}$ and every $x_{m}$ in $X$ with $x_{k}>$ $x_{j}>x_{i}$, we have $\operatorname{sgn}\left(v\left(x_{j}, x_{m}\right)\right) \geq \min \left\{\operatorname{sgn}\left(v\left(x_{k}, x_{m}\right)\right), \operatorname{sgn}\left(v\left(x_{i}, x_{m}\right)\right)\right\}$. There are two cases to check.

Case 1: Suppose $x_{m}>x_{j}$. It implies that $x_{m} \succeq x_{j}$, so we have $\operatorname{sgn}\left(v\left(x_{j}, x_{m}\right)\right) \leq 0$. Case 1.1: Suppose that $v\left(x_{j}, x_{m}\right)=0$. Since $x_{m} \succeq x_{i}$, we have $\operatorname{sgn}\left(v\left(x_{i}, x_{m}\right)\right) \leq 0$. Case 1.2: Suppose that $\operatorname{sgn}\left(v\left(x_{j}, x_{m}\right)\right)=$ -1 , that is, $x_{m} \succ x_{j}$. By transitivity, it implies $x_{m} \succ x_{i}$, so we have $\operatorname{sgn}\left(v\left(x_{i}, x_{m}\right)\right)=-1$.

Case 2: Suppose that $x_{j}>x_{m}$. It implies that $x_{j} \succeq x_{m}$, that is, $\operatorname{sgn}\left(v\left(x_{j}, x_{m}\right)\right) \geq 0$. Case 2.1: Suppose that $v\left(x_{j}, x_{m}\right)=0$. Since $x_{m} \succeq x_{i}$, $\operatorname{sgn}\left(v\left(x_{i}, x_{m}\right)\right) \leq 0$. Case 2.2: If $\operatorname{sgn}\left(v\left(x_{j}, x_{m}\right)\right)=1$, the inequality is satisfied because it is the largest value of the sign function.

Corollary 5. If a two-person symmetric zero-sum game has a generalized ordinal potential, then it is sign-quasiconcave. 
Chapter 3. Existence of pure equilibrium in two-person symmetric zero-sum games

$\begin{gathered}x \\ y \\ z \\ w \\ w\end{gathered}\left(\begin{array}{cccc}0 & -3 & -1 & -5 \\ 3 & 0 & 6 & 3 \\ 5 & -6 & 0 & 2 \\ 1 & -2 & 0\end{array}\right), \quad \begin{aligned} & y \\ & z \\ & x\end{aligned}\left(\begin{array}{cccc}0 & 1 & 1 & 1 \\ -1 & 0 & 1 & 1 \\ -1 & -1 & 0 & 1 \\ -1 & -1 & -1 & 0\end{array}\right)$

Figure 3.1: Games $(X, v)$ and $(X, \operatorname{sgn}(v))$ respectively

Proof. By Observation 8, if a two-person symmetric zero-sum game has a generalized ordinal potential, then the relation $\succeq$ is transitive in its equivalent decision problem. Hence Proposition 10 applies.

\subsection{Examples}

There are several elements of the results worth discussing in greater depth. Consider the two-person symmetric zero-sum game $(X, v)$ in Figure 3.1, in which only Player 1's payoff matrix is given. The rows and columns are labeled by the actions $x, y, z$ and $w$. Notice that the payoff function of the game $(X, \operatorname{sgn}(v))$ is the sign function of $v$ and that the place of the actions has been changed with respect to the game on the left.

Observe that the strategy $y$ guarantees the payoff of zero (the value of the game), which makes it an optimal strategy and $(y, y)$ a pure equilibrium for both games. However, this game does not appear to satisfy any sufficient condition but the sign-quasiconcavity. To see this, consider the sign function of $v$ and order the actions as $y, z, w, x$, which is given on the right in Figure 3.1. In this game, each column has a single peak, which makes the game $(X, v)$ sign-quasiconcave. By Proposition 9, we conclude that $(X, v)$ has a pure equilibrium.

First, notice that the game $(X, v)$ is not quasiconcave, because, no matter how the actions are ordered, it is not possible to obtain a matrix such that each column has a single-peak. Second, since we have $z \succ w \succ$ 


$$
\begin{gathered}
x \\
y \\
z \\
w
\end{gathered}\left(\begin{array}{cccc}
0 & 0 & 1 & 1 \\
0 & 0 & 1 & 1 \\
-1 & -1 & 0 & -1 \\
-1 & -1 & 1 & 0
\end{array}\right), \quad \begin{gathered}
x \\
y \\
z \\
w
\end{gathered}\left(\begin{array}{cccc}
30 & 30 & 25 & 15 \\
30 & 30 & 20 & 10 \\
25 & 20 & 0 & 5 \\
15 & 10 & 5 & 0
\end{array}\right)
$$

Figure 3.2: A game (left) and its generalized ordinal potential (right)

$x$ and $v(z, x)<\min \{v(z, w), v(w, x)\}$, by Observation 8 , the game $(X, v)$ cannot have a generalized ordinal potential.

It follows by definition that sign-quasiconcavity is invariant under multiplication of a particular payoff by a positive constant. For example, multiply $v(w, x)$ by $\frac{1}{5}$ which equals 1 and set $v(x, w)=-1$, keeping the symmetry property of the game in Figure 3.1. Then, the game $(X, v)$ would have a generalized ordinal potential but it would still fail to be quasiconcave. The generalized ordinal potential function is given by:

$$
\begin{aligned}
& x \\
& y \\
& z \\
& w
\end{aligned}\left(\begin{array}{cccc}
1 & 14 & 6 & 3 \\
15 & 16 & 12 & 13 \\
8 & 10 & 9 & 7 \\
2 & 11 & 5 & 4
\end{array}\right)
$$

The game in Figure 3.2 shows that a generalized ordinal potential game does not necessarily satisfy the condition in Proposition 8 . On the other hand, quasiconcavity does not imply this condition, either. The game below is clearly quasiconcave but it does not satisfy the Proposition 8 condition because the preferences in the equivalent decision problem are not transitive.

$$
\begin{aligned}
& x \\
& y \\
& z
\end{aligned}\left(\begin{array}{ccc}
0 & 0 & 0 \\
0 & 0 & 1 \\
0 & -1 & 0
\end{array}\right)
$$


Chapter 3. Existence of pure equilibrium in two-person symmetric zero-sum games

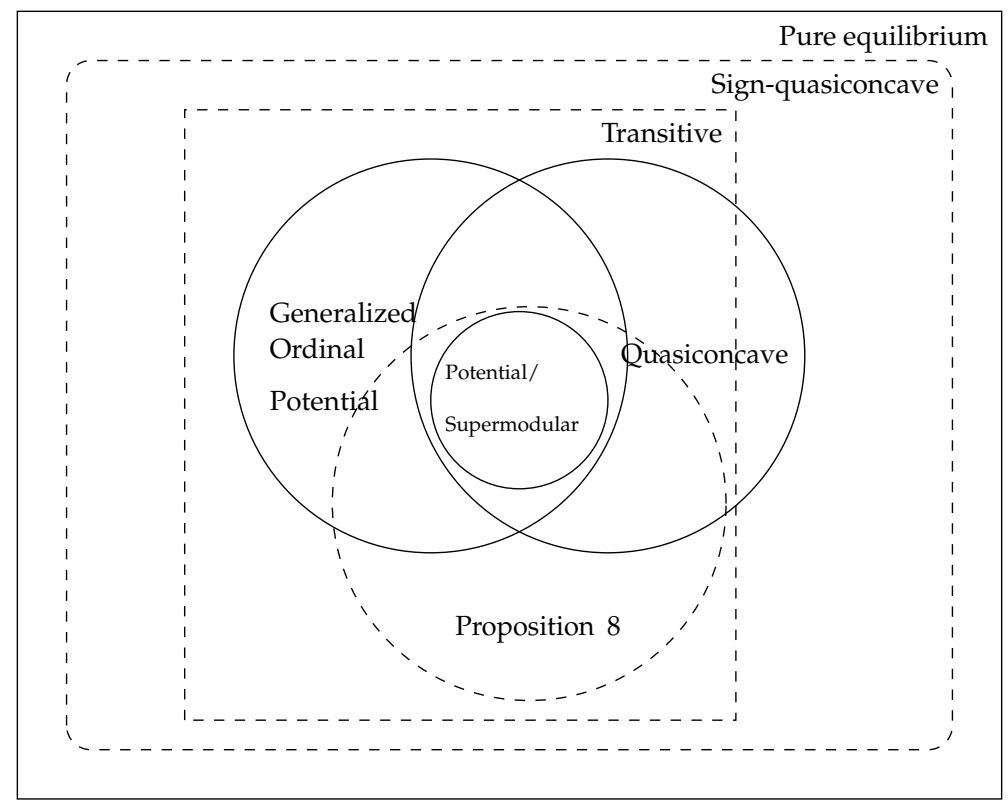

Figure 3.3: Relations between sufficient conditions on pure equilibria in twoperson symmetric zero-sum games

\subsection{Conclusion}

In this chapter, I have introduced three sufficient conditions for the existence of pure Nash equilibrium in two-person symmetric zero-sum games. I have obtained these conditions with the help of equivalent decision problems of the symmetric zero-sum games.

The so-called sign-quasiconcavity turned out to be the most general sufficient condition. I have showed that two-person symmetric zerosum games that possess this property have at least one equilibrium in pure strategies. Furthermore, this concept generalizes both generalized ordinal potentials (Monderer and Shapley, 1996) and quasiconcavity (Duersch et al., 2012a).

Figure 3.3 shows the inclusion relationships between several proper40 
ties in two-person symmetric zero-sum games, including the sufficient conditions proposed in this chapter, which are depicted by dashed lines. ${ }^{4}$ More specifically, I have showed that the equivalent decision problem of generalized ordinal potential games is transitive (Observation 8). Moreover, if the equivalent decision problem of a game is transitive, then it is a sign-quasiconcave game (Proposition 10). I have also showed that all quasiconcave games are sign-quasiconcave (Observation 7), but the reverse implication does not hold. Finally, I have proved that sign-quasiconcave games possess a Nash equilibrium in pure strategies (Proposition 9). ${ }^{5}$

\footnotetext{
${ }^{4}$ Note that the sizes of the shapes in Figure 3.3 are drawn arbitrarily.

${ }^{5}$ Note that Duersch et al. (2012a) showed that potential games and supermodular games (Topkis, 1998) coincide in two-person symmetric zero-sum games.
} 



\section{Chapter 4}

\section{Optimin equilibrium: An extension of maximin strategies}

\subsection{Introduction}

Economic interactions are not typically zero-sum, yet maximin strategies cannot capture mutual gains and losses in these interactive situations. A maximin strategist chooses an action to maximize the minimum utility he might receive under any conceivable deviation by the other player, "even assuming that his opponent is guided by the desire to inflict a loss rather than to achieve a gain" (von Neumann and Morgenstern, 1944, p. 555). This assumption leads to the following question: What happens when players act cautiously but do not harm themselves for the sake of harming others?

In this chapter, I capture cautious and self-interested behavior by assuming that players satisfy an individual rationality constraint and incorporate it with the maximin principle. Indeed, the resulting concept, optimin equilibrium, without this constraint reduces to a profile of maximin strategies in $n$-person noncooperative games. 
Chapter 4. Optimin equilibrium: An extension of maximin strategies

Von Neumann and Morgenstern (1944, p. 540) admit that the advantage of a player does not necessarily mean the disadvantage of other players and that their approach can be questioned by disregarding most economic and social interactions. These interactions include mutually beneficial trade, mutually harmful escalation, win-win situations, and positive and negative externalities created in an economy. Because maximin strategists play any game as if it is a zero-sum game, they ignore other players' utilities (i.e., preferences). These arguments call for a revision of the maximin strategy concept in nonstrictly competitive games.

The maximin strategy resolves the strategic uncertainty in a game in two steps: evaluation and comparison. The inner operator min represents the evaluation of strategies while the outer operator max represents a comparison across those evaluations. Similarly, optimin equilibrium employs the two steps as follows:

1. Evaluation: The value of a strategy profile is defined as the minimum utility players each receive under the others' individual rationality constraint-that rules out nonprofitable deviations (contribution of the chapter).

2. Comparison: The resulting multi-variable value function is optimized by applying Pareto optimality.

Accordingly, a strategy profile is called an optimin equilibrium if its value is Pareto optimal. Notice that Pareto optimality applies only to the value function, not to the utility function of the players. Furthermore, there is also no logical relationship between Pareto optimality and optimin equilibrium. I next present some games to illustrate the similarities and differences between optimin equilibrium, maximin strategy, Pareto optimality, and the benchmark solution, Nash equilibrium.

For example, in the battle of the sexes game, optimin equilibriumlike the persistent equilibrium (Kalai and Samet, 1984)—rejects the mixed 
strategy equilibrium, selecting only the two Pareto optimal profiles. This solution relates to the property that strong Nash equilibria (Aumann, 1959) form a subset of optimin equilibria.

However, an optimin equilibrium can be Pareto dominated. For example, in the one-shot prisoner's dilemma, each player defects in the unique optimin equilibrium, which is Pareto dominated. Nevertheless, optimin equilibrium may differ from Nash equilibrium. To illustrate the difference, consider the finitely repeated prisoner's dilemma with the following stage game:

\begin{tabular}{|c|c|c|}
\hline \multirow[b]{2}{*}{ Cooperate } & Cooperate & Defect \\
\hline & 3,3 & 0,5 \\
\hline Defe & 5,0 & 1,1 \\
\hline
\end{tabular}

The Tit-for-Tat strategy profile-in which a player begins by cooperating in the first round and then does what the opponent did in the previous round-turns out to be an optimin equilibrium which is not a Nash equilibrium. This prediction is surprising because the unique optimin equilibrium is (Defect, Defect) in the one-shot game but it disappears when the game is repeated. This is because, in the repeated game always playing Defect, for example, is not a profitable deviation from the Titfor-Tat profile and, therefore, is ruled out by the individual rationality constraint. Thus, the Tit-for-Tat guarantees a sufficiently high utility provided that the opponent is not guided by the desire to harm himself or herself. This property of the Tit-for-Tat was observed in some actual tournaments. ${ }^{1}$

To be sure, the value of an optimin equilibrium is only guaranteed under the individual rationality constraint of the opponent. Thus, if the

\footnotetext{
${ }^{1}$ Robert Axelrod conducted a tournament in 1979 in which computer programs played the repeated prisoner's dilemma with the above stage game (Axelrod, 1984). The Tit-for-Tat submitted by Anatol Rapoport was not only the simplest strategy, but it won the tournament. In a follow-up tournament, the Tit-for-Tat became the winner again-even though everyone knew the previous winner.
} 
Chapter 4. Optimin equilibrium: An extension of maximin strategies

opponent's goal is to inflict a loss rather than to act under this constraint, playing an optimin equilibrium strategy may then result in an unexpectedly low utility. This possibility suggests that optimin equilibrium predictions should be taken with a grain of salt in such situations.

In addition, I will illustrate some games in which optimin equilibrium arguably gives additional insights that are not captured by the benchmark solution, Nash equilibrium. In the so-called Inheritance Game, for example, the Nash equilibrium is the profile in which each strategy is weakly dominated by any other strategy, and the optimin equilibrium is the profile in which each strategy is the only undominated strategy in the game (see Example 1).

Moreover, I also show that the optimin value coincides with the maximin value in two-person zero-sum games. This result implies that the maximin solution, the Nash equilibrium, and the optimin equilibrium all select the mixed strategy profile $\left[\left(\frac{1}{3}, \frac{1}{3}, \frac{1}{3}\right),\left(\frac{1}{3}, \frac{1}{3}, \frac{1}{3}\right)\right]$ in the Rock-PaperScissors game.

In this chapter, I focus on the evaluation of strategic uncertainty in games, rather than the learning process or the epistemic conditions, which I leave for future research. In this regard, a related paper is Aumann and Dreze (2008) in which the authors study what players can rationally expect from the strategic uncertainty in a game. Despite starting with completely different assumptions, I do obtain similar conclusions to theirs, including: (1) the optimin value coincides with the maximin value in two-person zero-sum games, and (2) there are usually multiple values in $n$-person games (e.g., battle of the sexes game). Another related paper is Puppe and Schlag (2009) in which the authors show that the axioms of Milnor (1954) that characterize the maximin decision rule (Wald, 1950) are consistent with ignoring some states in which all payoffs are "small." This approach differs from the optimin approach in that-like maximin strategies-it does not take other players' preferences into account when 
4.2. Definition of optimin equilibrium

applied to strategic games.

This chapter is organized as follows. In Section 4.2, I define optimin equilibrium and prove its existence in strategic games with compact strategy sets and continuous utility functions. In Section 4.3, I discuss optimin equilibrium predictions in a selection of classical games, including some of its most important properties. Finally, in Section 4.4, I apply optimin equilibrium to the Arrow-Debreu economy.

\subsection{Definition of optimin equilibrium}

Let $\left(\Delta X_{i}, u_{i}\right)_{i \in N}$ be a two-person game in mixed extension, where $N=$ $\{1, \ldots, n\}$ is the finite set of players, $\Delta X_{i}$ the set of all probability distributions over the finite action set $X_{i}$, and $u_{i}: \Delta X_{1} \times \Delta X_{2} \rightarrow \mathbb{R}$ the von Neumann-Morgenstern expected utility function of player $i \in N$. For simplicity, assume $n=2$. (The results in this chapter do not depend on $n$ unless otherwise specified.) The notation $p \in \Delta X_{1} \times \Delta X_{2}$ represents a strategy profile. $^{2}$

Von Neumann and Morgenstern (1944, p. 11) argued that a game is an uncertainty which cannot be resolved by statistical assumptions. As I mentioned in the Introduction, the maximin strategy method for resolving this uncertainty can be decomposed into two steps: evaluation and comparison.

For example, consider a two-person game played by Alice and Bob. As for Alice, the uncertainty in playing a strategy $x_{1} \in X$ is that she does not know what Bob will choose. First, this uncertainty is evaluated by calculating the minimum utility with respect to all conceivable deviations by Bob (because he has a desire to inflict a loss). Second, these evaluations of strategies are compared by maximizing with respect to all strategies.

\footnotetext{
${ }^{2}$ For a detailed discussion of the mixed strategy concept, see Luce and Raiffa (1957, p. 74). For a more recent discussion, see Rubinstein (1991). As is standard in game theory, I assume that what matters is the consequence of strategies (consequentialist approach) so that I can define the utility functions over the strategy profiles.
} 
Chapter 4. Optimin equilibrium: An extension of maximin strategies

This yields a unique evaluation $V$ for the whole game-the maximin value of the game. In other words, the maximum utility that Alice can guarantee under the uncertainty of playing this game is $V$. She might receive a greater utility than $V$ by playing an optimal strategy, but she expects the worst outcome. ${ }^{3}$

By definition, nonzero-sum games differ from zero-sum games in that they allow mutually beneficial and harmful outcomes. To capture this property, I assume that Bob does not 'harm' himself for the sake of harming Alice. Formally, a strategy $p_{i}^{\prime} \in \Delta X_{i}$ is said to be a profitable deviation for player $i$ with respect to the profile $\left(p_{i}, p_{j}\right)$ if $u_{i}\left(p_{i}^{\prime}, p_{j}\right)>$ $u_{i}\left(p_{i}, p_{j}\right)$.

Definition 6. The individual rationality constraint, $I R C_{i}(p)$, of player $i$ at the profile $p$ consists of $p_{i}$ and the set of profitable deviations from $p$.

This is surely not the only way of restricting a player's deviations. But I propose a simple extension to the current setting by allowing deviations that are profitable, providing a context that can be further studied. ${ }^{4}$

I next define Alice's evaluation of a profile $p$ as the minimum utility she would receive under Bob's individual rationality constraint.

Definition 7. The value function $v: \Delta X_{1} \times \Delta X_{2} \rightarrow \mathbb{R} \times \mathbb{R}$ is defined as

$$
v_{i}(p)=\inf _{p_{j}^{\prime} \in I R C_{j}(p)} u_{i}\left(p_{i}, p_{j}^{\prime}\right)
$$

where $v_{i}$ denotes the $i^{\prime}$ th component of $v$ for $i \in\{1,2\}$ and $i \neq j$.

\footnotetext{
${ }^{3}$ Formally, Alice's evaluation $V_{x_{1} x_{2}}$ of the strategy $x_{1}$ in $\left(x_{1}, x_{2}\right)$ is given by $V_{x_{1} x_{2}}=$ $\min _{p_{2} \in \Delta X_{2}} u_{1}\left(x_{1}, p_{2}\right)$. Note that for all $x_{2}$ the evaluation of the profile $\left(x_{1}, x_{2}\right)$ is the same, that is $V_{x_{1} x_{2}}=V_{x_{1} p_{2}}$ for all $p_{2} \in \Delta X_{2}$. Thus, it is possible to assign a unique evaluation $V_{p_{1}}$ for every strategy $p_{1} \in \Delta X_{1}$. These evaluations are then compared by taking the maximum of $V_{p_{1}}$ with respect to $p_{1}$. Accordingly, the value of the game is defined by $V=\max _{p_{1} \in \Delta X_{1}} V_{p_{1}}$.

${ }^{4}$ This constraint is reminiscent of the individual rationality constraint used, for example, in contract theory in the sense that individually rational behavior always yields greater or equal utility than individually nonrational behavior.
} 
4.2. Definition of optimin equilibrium

$\underset{0}{\stackrel{\text { Alice chooses from }}{\longrightarrow}}$

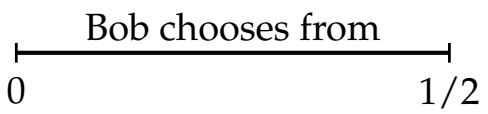

Figure 4.1: Inheritance Game

Unlike in maximin strategies, the value function does not assign a unique value to every strategy of Alice. ${ }^{5}$ Instead, the evaluation of uncertainty is attached to the strategy profile, like in the value notion of Nash (1951). ${ }^{6}$ To illustrate, I explore the Inheritance Game.

Example 1 (Inheritance Game). A couple delegates a person, $D$, to divide a cake (e.g., their inheritance) of unit length among their children, Alice and Bob. Players are in separate rooms and each have half of the cake on the table in front of them. By cutting, each chooses a piece from the cake, [0,1/2], which is illustrated in Figure 4.1. The rules of the noncooperative game are as follows:

1. If some of the players disagree with the rules, nobody will receive anything. The choice 0 expresses the disagreement.

2. Otherwise, each receives their own piece. In addition, if there is some piece left from either player, $D$ will pay each an extra 1 unit of money (as he would like to taste the cake and convince them to agree with this rule).

Notice that Alice and Bob each receive half of the cake at the profile $(1 / 2,1 / 2)$. According to the maximin strategy concept, the evaluation of the strategy $1 / 2$ for Alice is 0 . This is because, Bob may deviate to the strategy 0 , leaving Alice with a payoff of 0 . However, this deviation is

\footnotetext{
${ }^{5}$ To extend the definition of value function to $n$-person games, replace " $j$ " with " $-i$ " in Definition 7 , where $I R C_{-i}(p)$ denotes the union of all $I R C$ s of every player but $i$.

${ }^{6}$ Nash (1951, p. 291) defines the value of a game to a player as the payoff that the player receives from a Nash equilibrium when all the Nash equilibria payoffs are the same for that player. The value function assigns a value to each strategy profile, including the Nash equilibria. In particular, when a strategy profile is a Nash equilibrium, the value of a player at this profile is equal to her Nash equilibrium payoff, because there is no unilateral profitable deviation from it.
} 
Chapter 4. Optimin equilibrium: An extension of maximin strategies

not in Bob's interest since he will also receive 0. Because such deviations violate the IRC of the deviator, they are ruled out in the evaluation of a strategy profile.

By comparison, the value of the profile $(1 / 2,1 / 2)$ is $1 / 2$ for each according to the value function. This is because, each player guarantees receiving half of the cake at this profile under any profitable deviation of the other. (Notice that playing 0 is not a profitable deviation from this profile.)

The next step is to make comparisons among the evaluations of the strategy profiles. The multi-variable value function is optimized by applying Pareto optimality. Accordingly, a profile is an optimin equilibrium if its value is Pareto optimal. Formally, let $\left(\Delta X_{1}, \Delta X_{2}, u_{1}, u_{2}\right)$ be a two-person game and $v=\left(v_{i}, v_{j}\right)$ be the value function of the game.

Definition 8. A strategy profile $p$ is called optimin equilibrium if for every player $i \neq j$ and every $p^{\prime}, v_{i}\left(p^{\prime}\right)>v_{i}(p)$ implies $v_{j}\left(p^{\prime}\right)<v_{j}(p)$.

Unfortunately, it is not possible to refer to a strategy in an optimin equilibrium in the same spirit of a maximin strategy because a strategy in this setting makes sense only as part of a strategy profile. Notice that Pareto optimality applies only to the value function, not to the utility function of the players. Furthermore, there is also no logical relationship between Pareto optimality and optimin equilibrium.

In the battle of the sexes game illustrated below, for example, the two optimin equilibria are (Football, Football) and (Opera, Opera), which are Pareto optimal.

\begin{tabular}{r|c|c|}
\multicolumn{1}{c}{} & \multicolumn{1}{c}{ Football } & Opera \\
\cline { 2 - 3 } Football & 2,1 & 0,0 \\
\cline { 2 - 3 } Opera & 0,0 & 1,2 \\
\cline { 2 - 3 } & &
\end{tabular}

However, optimin equilibrium may be Pareto dominated. In the prisoner's dilemma, the unique optimin equilibrium is (Defect, Defect), 
which is Pareto dominated.

\begin{tabular}{|c|c|c|}
\hline & Cooperate & Defect \\
\hline at & 3,3 & 0,5 \\
\hline Defect & 5,0 & 1,1 \\
\hline
\end{tabular}

Optimin equilibrium may arguably give additional insights that are not captured by Nash equilibrium in some games. To illustrate, I return to the Inheritance Game. The unique Nash equilibrium is $(0,0)$ because at any other profile a player can profitably deviate by claiming higher, unless the other claims $1 / 2$ in which case the player can profitably deviate to slightly below $1 / 2$ to get the additional 1 unit. This also implies that $1 / 2$ is not a dominant strategy. Moreover, every strategy is rationalizable (Bernheim, 1984; Pearce, 1984) because it is a best response to 0 , which is rationalizable.

This game resembles a Bertrand duopoly because 0 is not only a weakly dominated strategy but it is dominated by any strategy. However, unlike in a duopoly game, all strategies but $1 / 2$ are dominated because $a^{\prime}$ dominates $a$ whenever $1 / 2>a^{\prime}>a$. Furthermore, the claim $1 / 2$ is not dominated because no other strategy guarantees half of the cake except when the opponent chooses 0 . As a result, the strategy $1 / 2$ is the only undominated strategy for each player.

Consider the profile $(1 / 2,1 / 2)$. Each player has a profitable deviation from it but the deviator would receive a smaller piece from the cake than the nondeviator, which gives incentives to free ride on the deviators. Thus, each player prefers the situation in which the other player deviates from this profile, which is a social dilemma that resembles a public goods contribution problem (for a related problem, see Bliss and Nalebuff, 1984). To compare and contrast with the Inheritance Game, consider the Guessing Game.

Example 2 (Guessing Game). Each of $n$ players chooses a number from the 
Chapter 4. Optimin equilibrium: An extension of maximin strategies

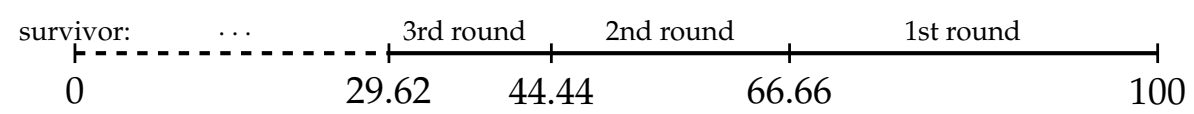

Figure 4.2: Rounds of iterated elimination of dominated strategies in the guessing game

interval $[0,100]$. The winner is the player whose guess is closest to the $2 / 3$ of the average of the chosen numbers. If there is more than one winner, the prize is shared equally among them.

Because choosing any number above approximately 66.66 is weakly dominated, it will be eliminated in the first round by the iterated elimination of weakly dominated strategies (see Figure 4.2). In the second round, any number above approximately 44.44 will be eliminated, and so on. The only surviving strategy after the iterated elimination of weakly dominated strategies is 0 .

As is well-known, the unique Nash equilibrium is the profile in which everyone plays 0 , which is also the unique optimin equilibrium. This is because, first, if the average is positive, some players can profitably deviate and earn the prize, which leaves 0 to the others, and, second, if the average is zero, each receives a positive payoff, which makes it the optimin equilibrium.

A difference between the Guessing Game and the Inheritance Game is that the strategy 0 is the only strategy that survives the iterated elimination of weakly dominated strategies in the former game, whereas it is the only strategy that is dominated by all other strategies in the latter. Thus, playing 0 in the Inheritance Game is comparable to playing a number above 66.66 in the Guessing Game. 


\subsection{Definition of optimin equilibrium}

\subsubsection{Existence of optimin equilibrium}

The following lemma presents a property of the value function which will be used in the following existence result.

Lemma 3. The value function of a player is upper semi-continuous.

Proof. In several steps, I will show that the value function $v_{i}$ of player $i$ in a game $\Gamma=\left(\Delta X_{1}, \Delta X_{2}, u_{1}, u_{2}\right)$ is upper semi-continuous.

First, we decompose the value function as

$$
v_{i}(p)=\min \left\{\inf _{p_{j}^{\prime} \in B_{j}(p)} u_{i}\left(p_{i}, p_{j}^{\prime}\right), u_{i}(p)\right\},
$$

where $B_{j}(p)$ is the (strict) better response correspondence of player $j$ with respect to $p$, representing the set of profitable deviations, which is defined as $B_{j}(p)=\left\{p_{j}^{\prime} \in \Delta X_{j} \mid u_{j}\left(p_{i}, p_{j}^{\prime}\right)>u_{j}(p)\right\}$.

I next show that the correspondence $B_{j}: \Delta X_{i} \times \Delta X_{j} \rightarrow \Delta X_{j}$ is lower hemi-continuous. For this, it is enough to show the graph of $B_{j}$ defined as follows is open.

$$
\operatorname{Gr}\left(B_{j}\right)=\left\{\left(q, p_{j}\right) \in \Delta X_{i} \times \Delta X_{j} \times \Delta X_{j} \mid p_{j} \in B_{j}(q)\right\} .
$$

$\operatorname{Gr}\left(B_{j}\right)$ is open in $\Delta X_{i} \times \Delta X_{j} \times \Delta X_{j}$ if and only if its complement is closed. Let $\left[\left(p_{j}, q_{i}, q_{j}\right)^{k}\right]_{k=1}^{\infty}$ be a sequence in $\left[G r\left(B_{j}\right)\right]^{c}=\left(\Delta X_{i} \times \Delta X_{j} \times\right.$ $\left.\Delta X_{j}\right) \backslash G r\left(B_{j}\right)$, converging to $\left(p_{j}, q_{i}, q_{j}\right)$ where $p_{j}^{k} \notin B_{j}\left(q^{k}\right)$ for all $k$. That is, we have $u_{j}\left(p_{j}^{k}, q_{i}^{k}\right) \leq u_{j}\left(q^{k}\right)$ for all $k$. Continuity of $u_{j}$ implies that $u_{j}\left(p_{j}, q_{i}\right) \leq u_{j}(q)$ which means $p_{j} \notin B_{j}(q)$. Hence $\left[G r\left(B_{j}\right)\right]^{c}$ is closed, implying that $B_{j}$ is lower hemi-continuous.

Next, we define $\hat{u}_{i}: \Delta X_{i} \times \Delta X_{j} \times \Delta X_{j} \rightarrow \mathbb{R}$ by $\hat{u}_{i}\left(q_{i}, q_{j}, p_{j}\right)=u_{i}\left(p_{j}, q_{i}\right)$ for all $\left(q_{i}, q_{j}, p_{j}\right) \in \Delta X_{i} \times \Delta X_{j} \times \Delta X_{j}$. Since $u_{i}$ is continuous, $\hat{u}_{i}$ is also continuous. In addition, we define $\bar{u}_{i}: G r\left(B_{j}\right) \rightarrow \mathbb{R}$ as the restriction of $\hat{u}_{i}$ to $\operatorname{Gr}\left(B_{j}\right)$, that is $\bar{u}_{i}=\hat{u}_{i_{\mid G r\left(B_{j}\right)}}$. The continuity of $\hat{u}_{i}$ implies the continuity of its restriction $\bar{u}_{i}$, which in turn implies $\bar{u}_{i}$ is upper semi-continuous. 
Chapter 4. Optimin equilibrium: An extension of maximin strategies

By Theorem 1 of Berge (1959, p. 115), lower hemi-continuity of $B_{j}$ and lower semi-continuity of $-\bar{u}_{i}: G r\left(B_{j}\right) \rightarrow \mathbb{R}$ imply that the function $-\bar{v}_{i}$ : $\Delta X_{i} \times \Delta X_{j} \rightarrow \mathbb{R}$-defined as $-\bar{v}_{i}(q)=\sup _{p_{j} \in B_{j}(q)}-\bar{u}_{i}\left(p_{j}, q\right)$-is lower semi-continuous. ${ }^{7}$ It implies that the function $\bar{v}_{i}(q)=\inf _{p_{j} \in B_{j}(q)} \bar{u}_{i}\left(p_{j}, q\right)$ is upper semi-continuous. ${ }^{8}$

As a result, the value function $v_{i}(q)=\min \left\{\bar{v}_{i}(q), u_{i}(q)\right\}$ of player $i$ is upper semi-continuous, because the minimum of two upper semicontinuous functions is also upper semi-continuous.

The following theorem shows that optimin equilibrium exists in mixed strategies.

Theorem 1. Every mixed extension of a finite game has an optimin equilibrium.

Proof. Define $v_{i}^{\text {max }}=\arg \max _{q \in \Delta X_{i} \times \Delta X_{j}} v_{i}(q)$, which is a nonempty compact set because $\Delta X_{i} \times \Delta X_{j}$ is compact and $v_{i}$ is upper semi-continuous by Lemma 3. Since $v_{i}^{\max }$ is compact and $v_{j}$ is also upper semi-continuous, the set $v_{i j}^{\max }=\arg \max _{q \in v_{i}^{\max }} v_{j}(q)$ is nonempty and compact. Clearly, the profiles in $v_{i j}^{\max }$ are Pareto optimal with respect to the value function, implying that $v_{i j}^{\max }$ is a nonempty compact subset of the set of optimin equilibria in the game. Analogously, the set $v_{j i}^{\max }$ is also a nonempty compact subset of the set of optimin equilibria. (Note that these arguments can be applied to games with any finite number of players.)

Notice that I have used neither the convexity of the strategy sets nor the concavity of the utility functions in the proof of Lemma 3 or Theorem 1. Thus, the latter result can be stated more generally as follows: Any game with continuous utility functions and compact strategy spaces possesses an optimin equilibrium.

\footnotetext{
${ }^{7}$ I follow the terminology, especially the definition of upper hemi-continuity, presented in Aliprantis and Border (1994, p. 569).

${ }^{8}$ I use the fact that a function $f$ is lower semi-continuous if and only if $-f$ is upper semicontinuous.
} 
4.3. Properties and more examples

\subsection{Properties and more examples}

Harsanyi and Selten (1988, p. 70) argued that invariance with respect to positive linear transformations of the utilities is a fundamental requirement for a solution concept. This requirement is satisfied by optimin equilibrium as the next property shows.

Property 1. Optimin equilibria are invariant to positive linear transformation of the utilities.

Proof. Let $\Gamma$ and $\hat{\Gamma}$ be two games such that that $\hat{u}_{i}=\alpha u_{i}+\beta$ for some $\alpha>0$ and some constant $\beta$. First, we have that $\hat{v}_{i}=\alpha v_{i}+\beta$ because strict better response correspondence does not change, and we can take $\alpha$ and $\beta$ out of the infimum in the definition of $v_{i}$. Second, a profile $p$ is a Pareto optimal profile with respect to $v$ if and only if it is Pareto optimal with respect to $\hat{v}$, because each $v_{i}$ is a positive linear transformation of $\hat{v}_{i}$. As a result, the set of optimin equilibria of $\Gamma$ and $\hat{\Gamma}$ are the same.

If the individual rationality constraint is removed from the definition of the value function, then the maximin strategy solution is recovered as the following property shows.

Property 2. Optimin equilibrium with a value function not satisfying the IRC reduces to a profile of maximin strategies.

Proof. We take the infimum over $\Delta X_{j}$ instead of taking it over the IRC in the value function definition. Therefore, $\bar{v}_{i}(p)=\inf _{p_{j}^{\prime} \in \Delta X_{j}} u_{i}\left(p_{i}, p_{j}^{\prime}\right)$. Accordingly, $\bar{p}$ is a Pareto dominant profile of the value function $\bar{v}$ where $\bar{p}_{i} \in \arg \max _{p_{i}^{\prime} \in \Delta X_{i}} \inf _{p_{j}^{\prime} \in \Delta X_{j}} u_{i}\left(p_{i}^{\prime}, p_{j}^{\prime}\right)$. It is clear that $\bar{p}_{i}$ is a maximin strategy of player $i$.

Two-person zero-sum games are both historically and theoretically important class in game theory. I illustrate the relationship between the 
Chapter 4. Optimin equilibrium: An extension of maximin strategies

equilibrium solution of von Neumann (1928) and the optimin equilibrium in this class of games.

The following property shows that a strategy profile is an optimin equilibrium if and only if it is a pair of maximin strategies in zero-sum games.

Property 3. Let $\left(Y_{1}, Y_{2}, u_{1}, u_{2}\right)$ be a two-person zero-sum game where $Y_{i}$ is not necessarily finite. A profile $\left(y_{1}^{*}, y_{2}^{*}\right) \in Y_{1} \times Y_{2}$ is an optimin equilibrium if and only if $y_{1}^{*} \in \arg \max _{y_{1}} \inf _{y_{2}} u_{1}\left(y_{1}, y_{2}\right)$ and $y_{2}^{*} \in \arg \max _{y_{2}} \inf _{y_{1}} u_{2}\left(y_{1}, y_{2}\right)$.

Proof. ' $\Rightarrow$ ' First, we show that $v_{i}\left(y_{i}, y_{j}\right)=\inf _{y_{j}^{\prime} \in Y_{j}} u_{i}\left(y_{i}, y_{j}^{\prime}\right)$ for each $i \neq j$. Suppose that there exists $\bar{y}_{j} \in Y_{j}$ such that $\bar{y}_{j} \in \arg \min _{y_{j}^{\prime} \in Y_{j}} u_{i}\left(y_{i}, y_{j}^{\prime}\right)$. Then $v_{i}\left(y_{i}, y_{j}\right)=\min _{y_{j}^{\prime} \in Y_{j}} u_{i}\left(y_{i}, y_{j}^{\prime}\right)=u_{i}\left(y_{i}, \bar{y}_{j}\right)$. Suppose, otherwise, that for all $y_{j}^{\prime} \in Y_{j}$ there exists $y_{j}^{\prime \prime} \in Y_{j}$ such that $u_{i}\left(y_{i}, y_{j}^{\prime \prime}\right)<u_{i}\left(y_{i}, y_{j}^{\prime}\right)$. It implies that $v_{i}\left(y_{i}, y_{j}\right)=\inf _{y_{j}^{\prime}: u_{i}\left(y_{i}, y_{j}^{\prime}\right)<u_{i}\left(y_{i}, y_{j}\right)} u_{i}\left(y_{i}, y_{j}^{\prime}\right)=\inf _{y_{j}^{\prime} \in Y_{j}} u_{i}\left(y_{i}, y_{j}^{\prime}\right)$.

Next, we show that the value of an optimin equilibrium $\left(y_{1}^{*}, y_{2}^{*}\right)$ must be Pareto dominant in a zero-sum game. By contraposition, suppose that its value is not Pareto dominant; that is, there is another optimin equilibrium $\left(\hat{y}_{1}, \hat{y}_{2}\right)$ such that $v_{i}\left(y_{1}^{*}, y_{2}^{*}\right)>v_{i}\left(\hat{y}_{1}, \hat{y}_{2}\right)$ and $v_{j}\left(y_{1}^{*}, y_{2}^{*}\right)<$ $v_{j}\left(\hat{y}_{1}, \hat{y}_{2}\right)$ for $i \neq j$. Then, we have $v_{1}\left(y_{1}^{*}, y_{2}^{*}\right)=v_{1}\left(y_{1}^{*}, \hat{y}_{2}\right)$ and $v_{2}\left(\hat{y}_{1}, \hat{y}_{2}\right)=$ $v_{2}\left(y_{1}^{*}, \hat{y}_{2}\right)$. It implies that the value of $\left(y_{i}^{*}, \hat{y}_{j}\right)$ Pareto dominates the value of $\left(y_{1}^{*}, y_{2}^{*}\right)$ which is a contradiction to our supposition that $\left(y_{1}^{*}, y_{2}^{*}\right)$ is an optimin equilibrium. Since the value of $\left(y_{1}^{*}, y_{2}^{*}\right)$ is Pareto dominant, each strategy is a maximin strategy of the respective players.

' $\Leftarrow$ ' Suppose that for each $i$ we have $y_{i}^{*} \in \arg \max _{y_{i}} \inf _{y_{j}} u_{i}\left(y_{i}, y_{j}\right)$. It implies that for all $\left(y_{1}^{\prime}, y_{2}^{\prime}\right) \in Y_{1} \times Y_{2}$ and for each $i$ we have $v_{i}\left(y_{1}^{*}, y_{2}^{*}\right) \geq$ $v_{i}\left(y_{1}^{\prime}, y_{2}^{\prime}\right)$. Hence the value of $\left(y_{1}^{*}, y_{2}^{*}\right)$ is Pareto dominant which implies that it is an optimin equilibrium.

Corollary 6. In a zero-sum game, optimin equilibrium and Nash equilibrium coincide whenever a Nash equilibrium exists. 
4.3. Properties and more examples

This result implies that the optimin equilibrium coincides with the maximin solution and the Nash equilibrium at the mixed strategy profile $\left[\left(\frac{1}{3}, \frac{1}{3}, \frac{1}{3}\right),\left(\frac{1}{3}, \frac{1}{3}, \frac{1}{3}\right)\right]$ in the Rock-Paper-Scissors game illustrated below:

\begin{tabular}{r|c|c|c|}
\multicolumn{1}{c}{} & \multicolumn{1}{c}{ Rock } & Paper & \multicolumn{1}{c}{ Scissors } \\
\cline { 2 - 4 } Rock & 0,0 & $-1,1$ & $1,-1$ \\
\cline { 2 - 4 } Paper & $1,-1$ & 0,0 & $-1,1$ \\
\cline { 2 - 4 } Scissors & $-1,1$ & $1,-1$ & 0,0 \\
\cline { 2 - 4 } & &
\end{tabular}

Optimin equilibrium and equilibrium coincide in zero-sum games whenever the latter exists. However, optimin equilibrium may exist even though an equilibrium does not exist in which case it is a pair of maximin strategies. And the maximin value of a player coincides with his or her optimin value. The following property illustrates a relation between a refinement of Nash equilibrium and optimin equilibrium.

Property 4. A strong Nash equilibrium is always an optimin equilibrium.

Proof. The utility vector of a Nash equilibrium is the same as its value, because there is no unilateral profitable deviation from it. In addition, a strong Nash equilibrium (Aumann, 1959) is always Pareto optimal, which implies that it must be an optimin equilibrium.

For example, in the stag-hunt game below, the strong Nash equilibrium (Stag, Stag) is also the optimin equilibrium.

\begin{tabular}{c|c|c|}
\multicolumn{1}{c}{} & \multicolumn{1}{c}{ Stag } & Hare \\
\cline { 2 - 3 } Stag & 2,2 & 0,1 \\
\cline { 2 - 3 } Hare & 1,0 & 1,1 \\
\cline { 2 - 3 } & &
\end{tabular}

The following property shows the existence of optimin equilibrium in pure strategies in ordinal games in which the utilities of the players are ordinal rather than cardinal.

Property 5. Every finite ordinal game has an optimin equilibrium in pure strategies. 
Chapter 4. Optimin equilibrium: An extension of maximin strategies

\begin{tabular}{|c|c|c|c|}
\hline & Top & Middle & Bottom \\
\hline Top & 100,100 & 100,105 & 0,0 \\
\hline Middle & 105,100 & 95,95 & 0,210 \\
\hline Bottom & 0,0 & 210,0 & 5,5 \\
\hline
\end{tabular}

Figure 4.3: A game in which the unique optimin equilibrium is (Top, Top)

The proof of this property is trivial since there are finitely many strategies and players in finite ordinal games. As an example, consider the one-shot game illustrated in Figure 4.3.

Bob has a profitable deviation from the profile (Top, Top) to Middle but Alice would still receive 100 in this case. Because this is the only deviation that satisfies the IRC and the game is symmetric, the value of (Top, Top) is $(100,100)$. Also, consider the profile (Middle, Middle) from which Bob might unilaterally deviate to Bottom whereby he has strict incentives to do so. This deviation leaves Alice with a payoff of 0 , so the value of (Middle, Middle) is $(0,0) .{ }^{9}$

The value function is maximized at the profile (Top, Top), so it is the unique optimin equilibrium. According to the maximin strategy concept, the evaluation of the strategy Top, for example, would be 0 because a deviation of the opponent to Bottom leaves the nondeviator with a payoff of 0 . Clearly, all three strategies are maximin strategies, which guarantee 0 . Furthermore, there is a unique Nash equilibrium in this game, which is (Bottom, Bottom).

In another example, consider the finitely repeated prisoner's dilemma with the stage game shown below. Recall from Section 4.2 that the unique optimin equilibrium in the one-shot game is (Defect, Defect). However, new solutions emerge when the game is repeated. Note that at every Nash equilibrium each player defects in each round in a finitely repeated

${ }^{9}$ The remaining values are as follows: $v_{1}$ (Bottom, Top $)=(0,0), v_{1}$ (Middle, Top $)=(0,100)$, $v_{1}($ Bottom, Middle $)=(5,0)$ and $v_{1}($ Bottom, Bottom $)=(5,5)$. 
prisoner's dilemma.

\begin{tabular}{r|c|c|}
\multicolumn{1}{c}{} & \multicolumn{1}{c}{ Cooperate } & Defect \\
\cline { 2 - 3 } Cooperate & 3,3 & 0,5 \\
\cline { 2 - 3 } Defect & 5,0 & 1,1 \\
\cline { 2 - 3 } & &
\end{tabular}

Robert Axelrod conducted a computer tournament in 1979 on the repeated prisoner's dilemma with the above stage game, which was won with the Tit-For-Tat strategy submitted by Anatol Rapoport (Axelrod, 1980). A player who plays the Tit-For-Tat strategy starts by cooperating in the first round and then does what the opponent did in the previous round.

It turns out that the Tit-For-Tat strategy profile is an optimin equilibrium in this finitely repeated prisoner's dilemma. This is because, at every strategy profile, a player will have a profitable deviation in the last round, $k$, unless the other plays Defect in which case the player would have a profitable deviation in round $k-1$. This implies that each Tit-ForTat strategist guarantees $3(k-1)$-the payoff players each receive in the first $(k-1)$ rounds - under any profitable deviation by the opponent, and no other profile can guarantee strictly better. Thus, it is an optimin equilibrium, though it is possibly not unique.

The following property shows that optimin equilibrium coincides with maximin strategy and Nash equilibrium in one-person games, differing from the maximin (minimax) decision rule in decision theory. ${ }^{10}$

Property 6. Optimin equilibrium in one-person games is equivalent to expected utility maximization.

Imagine a one-player game in which the decision maker is to make a choice between several gambles. In that case, optimin equilibrium

\footnotetext{
${ }^{10}$ See Wald (1950) for maximin decision rule, and see Gilboa and Schmeidler (1989) for an axiomatization of it.
} 
Chapter 4. Optimin equilibrium: An extension of maximin strategies

reduces to expected utility maximization-the decision maker chooses the gamble with the highest expected utility. However, according to the maximin decision rule, a decision maker chooses the gamble that maximizes her utility with respect to the worst state of the world (i.e., the state with the minimum outcome) even if the probability assigned to it is very small.

\subsection{An application: Arrow-Debreu economy}

Arrow and Debreu (1954) define abstract economy as a game situation in which-unlike in a standard game-the strategy sets of players depend on the strategies chosen by other players. In an abstract economy, the players are consumers, producers, and the market participant who chooses prices. The strategy set of a player depends on the choices of the others because players have constraints such as the budget constraint of consumers, which depend on the price and, in turn, depend on the choices of players.

Let $\mathcal{E}=\left(Y_{i}, u_{i}, F_{i}\right)_{i \in N}$ be an abstract economy where $N$ denotes the set of players, $Y_{i}$ the strategy set, $u_{i}$ the utility function, and $F_{i}: Y_{-i} \rightarrow Y_{i}$ the feasibility correspondence of player $i \in N$, which gives player $i$ 's set of feasible strategies given the other players' strategies.

The following two lemmas will be used in the proof of the existence theorem.

Lemma 4. The value function of each player is upper semi-continuous.

The proof follows essentially the same steps as the proof of Lemma 3, therefore I do not reproduce it. (Because utility functions are continuous, the strict better reply correspondences have open graphs so Berge's aforementioned theorem applies.)

Lemma 5. The correspondence $\mathcal{F}(y)=\left\{y \in Y \mid y_{i} \in F_{i}\left(y_{-i}\right)\right.$ for all $\left.i\right\}$ is compact for all $y$. 
Proof. We first show that $\mathcal{F}(y)$ is closed where $y \in Y$. Take a sequence $y^{k}$ converging to $\bar{y}$ such that such that $y^{k} \in \mathcal{F}(y)$ for all $k$. It implies that $y^{k} \in G r\left(F_{i}\right)=\left\{y \in Y \mid y_{i} \in F_{i}\left(y_{-i}\right\}\right.$ for all $i$. Since $G r\left(F_{i}\right)$ is closed by our supposition and $y^{k}$ converges to $\bar{y}$, we have $\bar{y} \in G r\left(F_{i}\right)$ for every $i$. This implies that $\bar{y} \in \mathcal{F}(y)$. Thus, $\mathcal{F}(y)$ is compact because it is a closed subset of a compact set.

The following theorem shows the existence of optimin equilibrium under some topological assumptions on the primitives of an abstract economy.

Theorem 2. Let $\mathcal{E}=\left(Y_{i}, u_{i}, F_{i}\right)_{i \in N}$ be an abstract economy. If $Y_{i}$ is compact, $u_{i}$ is continuous, and $F_{i}$ has a closed graph, then the economy $\mathcal{E}$ has an optimin equilibrium.

The proof of Theorem 1 can be applied to prove this theorem with an addition that one maximizes the value function with respect to $\mathcal{F}$ (i.e., $\left.v_{i}^{\max }=\arg \max _{y \in \mathcal{F}(y)} v_{i}(y)\right)$.

Property 7. Competitive economic equilibrium is an optimin equilibrium.

This follows from the second fundamental theorem of welfare economics: A competitive equilibrium in an Arrow-Debreu model (i.e., a Nash equilibrium of the abstract economy) is Pareto optimal. Therefore, it is an optimin equilibrium. However, even if a competitive equilibrium does not exist, optimin equilibrium may exist in an abstract economy by Theorem 2. This is because, I do not assume convexity of strategy sets, quasi-concavity of utility functions, or continuity and convexity of feasibility correspondences in this theorem.

Surely, I only show the mere possibility that an optimin equilibrium exists when a competitive equilibrium does not. In addition, I said nothing about the conditions on consumer preferences and producer technologies in this case, which I leave for a future project. 
Chapter 4. Optimin equilibrium: An extension of maximin strategies

\subsection{Conclusion}

I have introduced a concept dubbed optimin equilibrium that extends maximin strategies to $n$-person nonstrictly competitive games in which mutual gains and losses are possible. This extension is based on incorporating an individual rationality constraint into the maximin principle, which rules out nonprofitable deviations by players. I showed that optimin equilibrium without this constraint reduces to a profile of maximin strategies in $n$-person games.

In two-person zero-sum games, Nash equilibrium, maximin strategies, and optimin equilibrium all coincide. For example, the unique mixed strategy equilibrium in the classical Rock-Paper-Scissors game is also the unique optimin equilibrium.

In addition, I have showed that an optimin equilibrium may or may not be Pareto optimal. For example, in the battle of the sexes game optimin equilibria are those profiles that are Pareto optimal. However, in the prisoner's dilemma, optimin equilibrium is the Pareto dominated profile in which both players defect.

In the finitely repeated prisoner's dilemma, I showed that new optimin equilibria emerge. For example, one of the optimin equilibria in the repeated game discussed in Section 4.3 consists of the well-known Tit-for-Tat strategies. A Tit-for-Tat strategist starts by cooperating in the first round and then does what the opponent did in the previous round.

I have also applied optimin equilibrium to the Arrow-Debreu economy and given sufficient conditions for the existence of optimin equilibrium in this setting. I showed that every competitive equilibrium is an optimin equilibrium but optimin equilibrium may exist in an economy even if a competitive equilibrium does not exist. The specific conditions on consumer preferences and producer technologies that guarantee an optimin equilibrium are yet to be studied.

In this chapter, I focused on the evaluation of strategic uncertainty in 
games, leaving several related questions unexplored such as how players actually get to an optimin equilibrium and what the epistemic conditions behind this concept are. In addition, the optimin approach can be applied to games with Knightian uncertainty or ambiguity, which is a natural area of application for maximin strategies.

When I consider the reduced-normal form of an extensive-form game, optimin equilibrium solutions seem to have forward induction reasoning rather than backward induction. So, another research direction opened up by this chapter is to extend the definition of optimin equilibrium to extensive-form games. This direction would yield the exploration of its relation to forward and backward induction reasoning. 



\section{Chapter 5}

\section{Is rationality a personal trait? A paradox}

\subsection{Introduction and setting}

In this chapter, I will show that there are games and decision problems in which a decision maker cannot be simultaneously rational in both. Put differently, the same person can be simultaneously rational and irrational, hence the paradox. Accordingly, I will also show that the so-called doubly linearity is a necessary and sufficient condition for a decision maker to be simultaneously rational in both situations. This condition restricts the social preferences such as inequality aversion.

Let $G=\left(\Delta X, \Delta Y, \mathcal{U}_{1}, \mathcal{U}_{2}\right)$ be a game in mixed extension in which players are called Alice and Bob. The sets $\Delta X$ and $\Delta Y$ denote the set of all probability distributions over the pure strategy set $X=\left\{x_{1}, x_{2}, \ldots, x_{m}\right\}$ of Alice and the pure strategy set $Y=\left\{y_{1}, y_{2}, \ldots, y_{n}\right\}$ of Bob, respectively. I assume that players are rational in the sense that their preferences in $G$ can be represented by a von Neumann-Morgenstern expected utility function $\mathcal{U}_{i}: \Delta X \times \Delta Y \rightarrow \mathbb{R}$ for $i \in\{1,2\}$. The function $\mathcal{U}_{i}$ is the bilinear extension of the ordinal utility function $\hat{\mathcal{U}}_{i}: X \times Y \rightarrow \mathbb{R}$ for $i \in\{1,2\}$.

Let $A_{m \times n}$ and $B_{m \times n}$ be two matrices whose elements $a_{i j}$ and $b_{i j}$ 
Chapter 5. Is rationality a personal trait? A paradox

$$
G=\begin{gathered}
L \\
R \\
R
\end{gathered}\left(\begin{array}{cc}
5,10 & 5,0 \\
5,0 & 5,10
\end{array}\right) \quad D=\begin{array}{r}
L \\
R\left(\begin{array}{ll}
5 & 5 \\
5 & 5
\end{array}\right)
\end{array}
$$

Figure 5.1: A game and its associated decision problem. Numbers represent (non-utility) monetary payoffs.

denote - non-utility - monetary payoffs of Alice and Bob, respectively. (I assume monetary payoffs for simplicity.) I assume that when Alice chooses $x_{i}$ and Bob chooses $y_{j}$, Alice receives $a_{i j}$ and Bob receives $b_{i j}$ units of money. Note that for a player like Alice, for example, $\mathcal{U}_{1}\left(x_{i}, y_{j}\right)$ is not necessarily equal to the corresponding monetary payoff $a_{i j}$ unless we assume that utility is linear in money or players have purely selfish preferences.

Next, let $D=\left(\Delta X, \Delta Y, u_{1}\right)$ be the decision problem of Alice that is associated with the game $G$-Alice's monetary payoffs in $D$ are identical to those in $G$. I assume that Alice is rational in $D$; that is, her preferences can be represented by a von Neumann-Morgenstern expected utility function $u_{1}: \Delta X \times \Delta Y \rightarrow \mathbb{R}$, which is the bilinear extension of the ordinal utility function $\hat{u}_{1}: X \times Y \rightarrow \mathbb{R}$.

The associated decision problem can be thought of as a one-person game version of $G$, in which Bob is replaced by an unconscious lottery choosing actions. So, there is no payoff for another player in the decision problem, because there is no other player. In other words, when the outcome is $\left(x_{i}, y_{j}\right)$, Alice receives $a_{i j}$ units of money, and that is all. Figure 5.1 illustrates a game and its associated decision problem.

Because Alice's payoffs in the game are completely identical to her payoffs in the associated decision problem, the difference in her preferences come from the fact that she plays the game against a human player, Bob, who also receives payoffs. For each pair $\left(a_{i j}, b_{i j}\right)$, define the 


\subsection{Introduction and setting}

function $\hat{v}_{1}$ as $\hat{v}_{1}\left(a_{i j}, b_{i j}\right):=\mathcal{U}_{1}\left(x_{i}, y_{j}\right)-u_{1}\left(x_{i}, y_{j}\right)$. The function $\hat{v}_{1}$ represents Alice's social preferences-the additional utility (or disutility) that Alice receives from the outcome that Bob receives $b_{i j}$ when she receives $a_{i j}$. The set of pairs of feasible expected monetary payoffs are defined as follows: $M=\left\{\left(a_{p q}, b_{p q}\right) \in \mathbb{R}^{2} \mid a_{p q}=p^{\top} A q\right.$ and $b_{p q}=p^{\top} B q$ for $(p, q) \in$ $\Delta X \times \Delta Y\}$. Then, I extend $\hat{v}_{1}$ to $M$ by defining $v_{1}: M \rightarrow \mathbb{R}$ as

$$
v_{1}\left(a_{p q}, b_{p q}\right)=\mathcal{U}_{1}(p, q)-u_{1}(p, q)
$$

Example 3. Figure 5.1 illustrates a game where Alice is (ex ante) inequality averse - she suffers a disutility from uneven expected payoff distributions, but does not suffer any disutility from even ones. For the sake of argument, suppose that $u_{1}(L, L)=u_{1}(R, L)=5$ in the decision problem, and that Alice incurs a utility loss of 1 both from the outcome $(5,10)$ and the outcome $(5,0)$ in the game, that is, $v_{1}(5,10)=v_{1}(5,0)=-1 .^{1}$ This implies that $\mathcal{U}_{1}(L, L)=$ $\mathcal{U}_{1}(R, L)=4$.

Now consider the profile $\left(\left(\frac{1}{2}, \frac{1}{2}\right), L\right)$ in which Alice mixes $50-50$ between $L$ and $R$, and Bob plays L. Since $\mathcal{U}_{1}$ and $u_{1}$ are $v N-M$ utility functions, we have $\mathcal{U}_{1}\left(\left(\frac{1}{2}, \frac{1}{2}\right), L\right)=4$ and $u_{1}\left(\left(\frac{1}{2}, \frac{1}{2}\right), L\right)=5$, which imply that $v_{1}(5,5)=-1$. However, this contradicts the assumption that Alice suffers no disutility in this case, that is, $v_{1}(5,5)$ should have been zero. ${ }^{2}$

This paradoxical situation occurs due to the assumption that Alice is rational both in the game and in the decision problem. To put it differently, assuming the rationality of Alice in one domain forces her to be irrational (i.e., a person who is not rational) in the other domain. To

\footnotetext{
${ }^{1}$ Note that, as defined earlier, the game is played by Alice and Bob, whereas there is only one player, Alice, in the decision problem.

${ }^{2}$ The same conclusion can be obtained without assuming any specific value for the $v$ function as follows. I have $v_{1}(5,10)=\mathcal{U}_{1}(L, L)-u_{1}(L, L), v_{1}(5,0)=\mathcal{U}_{1}(R, L)-u_{1}(R, L)$ and $v_{1}(5,5)=$ $\mathcal{U}_{1}\left(\left(\frac{1}{2}, \frac{1}{2}\right), L\right)-u_{1}\left(\left(\frac{1}{2}, \frac{1}{2}\right), L\right)$. Since $\mathcal{U}_{1}$ and $u_{1}$ are vN-M utility functions, the latter equation is equal to $\frac{1}{2} \mathcal{U}_{1}(L, L)+\frac{1}{2} \mathcal{U}_{1}(L, R)-\frac{1}{2} u_{1}(L, L)-\frac{1}{2} u_{1}(L, R)=\frac{1}{2} v_{1}(5,10)+\frac{1}{2} v_{1}(5,0)$. As a result, we have $v_{1}(5,5)=\frac{1}{2} v_{1}(5,10)+\frac{1}{2} v_{1}(5,0)$, which is in conflict with the supposition that Alice incurs a utility loss from outcomes $(5,10)$ and $(5,0)$.
} 
resolve this, the following restriction is placed on the social preferences function.

Definition 9. A function $v$ is called doubly linear if for all $\left(a_{p q}, b_{p q}\right) \in M$,

$$
v\left(a_{p q}, b_{p q}\right)=\sum_{i=1}^{m} \sum_{j=1}^{n} p_{i} q_{j} v\left(a_{i j}, b_{i j}\right)
$$

Although doubly linearity seems to be a strong condition, it neither implies nor is implied by bilinearity. ${ }^{3}$

The following theorem shows that doubly linearity of the social preferences function is a necessary and sufficient condition for a decision maker to be rational both in games and in the associated decision problems.

Theorem 3. Suppose that $u_{1}$ is a vN-M utility function. Then, $\mathcal{U}_{1}$ is a $v N-M$ utility function if and only if $v_{1}$ is doubly linear.

Proof. ' $\Rightarrow$ ' By definition of $v_{1}$, for each pair $\left(a_{p q}, b_{p q}\right)$ in $M$ we have

$$
\begin{gathered}
v_{1}\left(a_{p q}, b_{p q}\right)=\mathcal{U}_{1}(p, q)-u_{1}(p, q) \\
\stackrel{(1)}{=} \sum_{i=1}^{m} \sum_{j=1}^{n} p_{i} q_{j} \mathcal{U}_{1}\left(x_{i}, y_{j}\right)-\sum_{i=1}^{m} \sum_{j=1}^{n} p_{i} q_{j} u_{1}\left(x_{i}, y_{j}\right) \\
\stackrel{(2)}{=} \sum_{i=1}^{m} \sum_{j=1}^{n} p_{i} q_{j}\left(u_{1}\left(x_{i}, y_{j}\right)+v_{1}\left(a_{i j}, b_{i j}\right)\right)-\sum_{i=1}^{m} \sum_{j=1}^{n} p_{i} q_{j} u_{1}\left(x_{i}, y_{j}\right) \\
\stackrel{(3)}{=} \sum_{i=1}^{m} \sum_{j=1}^{n} p_{i} q_{j} v_{1}\left(a_{i j}, b_{i j}\right) .
\end{gathered}
$$

where Equation (1) is obtained by the assumption that $\mathcal{U}_{1}$ and $u_{1}$ are vN-M utility functions, and (2) is obtained by definition of $v_{1}$. Finally, cancelling out the terms we obtain (3).

\footnotetext{
${ }^{3}$ Note that a bilinear function is a function that is linear in each argument. Clearly, a doubly linear function is not always linear in each argument.
} 
$' \Leftarrow$ ' By definition of $v_{1}$ we have $\mathcal{U}_{1}(p, q)=u_{1}(p, q)+v_{1}\left(a_{p q}, b_{p q}\right)$.

$\stackrel{(4)}{=} \sum_{i=1}^{m} \sum_{i=1}^{n} p_{i} q_{j} u_{1}\left(x_{i}, y_{j}\right)+\sum_{i=1}^{m} \sum_{j=1}^{n} p_{i} q_{j} v_{1}\left(a_{i j}, b_{i j}\right) \stackrel{(5)}{=} \sum_{i=1}^{m} \sum_{i=1}^{n} p_{i} q_{j} \mathcal{U}_{1}\left(x_{i}, x_{j}\right)$.

where (4) is obtained by using the supposition that $u_{1}$ is a vN-M utility function and $v_{1}$ is doubly linear. By definition of $v_{1}$, we obtain (5).

Corollary 7. If a player is rational in a game, then she cannot be rational in the associated decision problem unless her social preference function satisfies doubly linearity.

Diamond (1967) showed by an example that ex ante egalitarianism may not be consistent with Harsanyi's ex ante utilitarianism. Example 1 shares a similarity with Diamond's example in the sense that it uses the assumption of ex ante inequality aversion, though it reaches a different conclusion. The condition in Theorem 1 characterizes the social preferences of a player so that the same person-without making any interpersonal utility comparison-is simultaneously rational and irrational. For example, this condition not only rules out inequality aversion, but it also seems to rule out other social preferences including altruism.

\subsection{An example and conclusion}

The existence of mixed strategy equilibrium in a game might be in conflict with the rationality of a player in the associated decision problem. The game in Figure 5.2 illustrates this situation. Assume that Alice is ex ante inequality averse (as in Example 3) and that Bob is self-regarding. In addition, if we assume that Alice is rational in the associated decision problem, then the game does not possess a Nash equilibrium; if we assume that it has a Nash equilibrium, then Alice cannot be rational in the associated decision problem. 


$$
\begin{gathered}
L \\
T \\
M \\
B
\end{gathered}\left(\begin{array}{cc}
10,20 & 10,0 \\
10,0 & 10,40 \\
9,0 & 9,0
\end{array}\right)
$$

Figure 5.2: A game without a Nash equilibrium (numbers represent non-utility monetary payoffs).

First, suppose that Alice is rational in the decision problem. Notice that there is no Nash equilibrium in which Bob plays a pure strategy, because if Bob plays $L$, the best response of Alice to $L$ denoted by $B R_{1}(L)$ is $\left(\frac{1}{2}, \frac{1}{2}, 0\right)$, but $B R_{2}\left(\left(\frac{1}{2}, \frac{1}{2}, 0\right)\right)$ is $R$. Similarly, $B R_{1}(R)$ is $\left(\frac{3}{4}, \frac{1}{4}, 0\right)$, but $B R_{2}\left(\left(\frac{3}{4}, \frac{1}{4}, 0\right)\right)$ is $L$.

To reach a contradiction, suppose that $p$ is a Nash equilibrium strategy of Alice. Then we have $\mathcal{U}_{2}(p, L)=\mathcal{U}_{2}(p, R)$, which implies $20 p_{1}=$ $40 p_{2}$ and $p_{2}=\frac{p_{1}}{2}$. Since probabilities sum to 1 , we obtain $p_{3}=1-p_{1}-$ $\frac{p_{1}}{2}=\frac{2-3 p_{1}}{2}$. Since $\frac{2-3 p_{1}}{2} \geq 0$, we have $p_{1} \leq \frac{2}{3}$. For all values $p_{1}<\frac{2}{3}$, Bob's unique best response is $R$, but we showed that there is no Nash equilibrium corresponding to this case. If $p_{1}=\frac{2}{3}$, then $p_{2}=\frac{1}{3}$. However, $\left(\frac{2}{3}, \frac{1}{3}, 0\right)$ is never a best response, because it forces the outcome to be inequitable as the $v$ function will be negative: no matter what Bob plays, the expected payoffs will be $\left(10, \frac{40}{3}\right)$. However, Alice can decrease Bob's payoff sufficiently by deviating to a strategy in which she puts some probability on $B$. For example, if Bob plays $\left(\frac{2}{3}, \frac{1}{3}\right)$, Alice's best response would be $\left(\frac{27}{37}, 0, \frac{10}{37}\right)$ whose expected payoffs would be approximately $(9.73,9.73)$

Second, one may construct a Nash equilibrium in this game by altering the utilities as desired in the associated decision problem, since we do not specify any particular type of irrationality of Alice in the decision problem. 
To conclude, say that the concept of rationality is a personal trait if a 'rational' person acts rationally in every situation she faces. Is rationality a personal trait, or is it a situation-specific concept? In this chapter, I have showed that a decision maker can be rational in a game but irrational in the associated decision problem, or vice versa. This finding seems to suggest that rationality is a situation-specific concept. A natural research direction would be to extend this chapter to $n$-person games. 



\section{Bibliography}

Aliprantis, C. and K. Border (1994). Infinite Dimensional Analysis: A Hitchhiker's Guide (1996, Third ed.). Springer, Berlin, Heidelberg.

Arrow, K. J. and G. Debreu (1954). Existence of an equilibrium for a competitive economy. Econometrica: Journal of the Econometric Society, 265-290.

Aumann, R. J. (1959). Acceptable points in general cooperative n-person games. In R. D. Luce and A. W. Tucker (Eds.), Contribution to the theory of game IV, Annals of Mathematical Study 40, pp. 287-324. University Press.

Aumann, R. J. and J. H. Dreze (2008). Rational expectations in games. American Economic Review 98(1), 72-86.

Axelrod, R. (1980). Effective choice in the prisoner's dilemma. The Journal of Conflict Resolution 24(1), 3-25.

Axelrod, R. (1984). The evolution of cooperation (2006, revised ed.). Basic books, New York, NY.

Berge, C. (1959). Espaces topologiques: Fonctions multivoques. Dunod.

Bergstrom, T. C. (1975). Maximal elements of acyclic relations on compact sets. Journal of Economic Theory 10(3), 403-404. 
Bibliography

Bernheim, B. D. (1984). Rationalizable strategic behavior. Econometrica 52(4), 1007-1028.

Bliss, C. and B. Nalebuff (1984). Dragon-slaying and ballroom dancing: The private supply of a public good. Journal of Public Economics 25(1), $1-12$.

Debreu, G. (1954). Representation of a preference ordering by a numerical function. In R. Thrall, C. Davis, and R. Coombs (Eds.), Decision Processes, pp. 159-165. John Wiley, New York, NY.

Diamond, P. A. (1967). Cardinal Welfare, Individualistic Ethics, and Interpersonal Comparison of Utility: Comment. Journal of Political Economy 75, 765.

Duersch, P., J. Oechssler, and B. C. Schipper (2012a). Pure strategy equilibria in symmetric two-player zero-sum games. International Journal of Game Theory 41, 553-564.

Duersch, P., J. Oechssler, and B. C. Schipper (2012b). Unbeatable imitation. Games and Economic Behavior 76(1), 88-96.

Fishburn, P. C. (1982). Nontransitive measurable utility. Journal of Mathematical Psychology 26(1), 31-67.

Fishburn, P. C. (1984). Dominance in SSB utility theory. Journal of Economic Theory 34(1), 130-148.

Fishburn, P. C. (1988). Nonlinear preference and utility theory. Johns Hopkins University Press, Baltimore.

Fishburn, P. C. and R. W. Rosenthal (1986). Noncooperative games and nontransitive preferences. Mathematical Social Sciences 12(1), 1-7.

Fudenberg, D. and D. K. Levine (2006). A dual-self model of impulse control. The American Economic Review 96(5), 1449-1476. 
Bibliography

Gilboa, I. and D. Schmeidler (1989). Maxmin expected utility with nonunique prior. Journal of Mathematical Economics 18(2), 141-153.

Harsanyi, J. C. and R. Selten (1988). A General Theory of Equilibrium Selection in Games. MIT Press, Cambridge, MA.

Jensen, N. E. (1967). An introduction to Bernoullian utility theory: I. utility functions. The Swedish Journal of Economics 69(3), 163-183.

Kalai, E. and D. Samet (1984). Persistent equilibria in strategic games. International Journal of Game Theory 13(3), 129-144.

Luce, R. and H. Raiffa (1957). Games and Decisions: Introduction and Critical Survey. Dover books on advanced mathematics. Wiley, New York, NY.

Milnor, J. (1954). Games against nature. In R. M. Thrall, C. H. Coombs, and R. L. Davis (Eds.), Decision Processes. John Wiley, New York, NY.

Monderer, D. and L. S. Shapley (1996). Potential games. Games and Economic Behavior 14(1), 124-143.

Nash, J. (1951). Non-cooperative games. Annals of Mathematics 54(2), 286-295.

Nash, J. (1953). Two-person cooperative games. Econometrica 21(1), 128-140.

Owen, G. (1968). Game Theory. Academic Press, California.

Pearce, D. G. (1984). Rationalizable strategic behavior and the problem of perfection. Econometrica 52(4), 1029-1050.

Potters, J. A., T. Raghavan, and S. H. Tijs (1999). Pure equilibrium strategies for stochastic games via potential functions. Katholieke Universiteit Nijmegen. Mathematisch Instituut. 
Puppe, C. and K. H. Schlag (2009). Choice under complete uncertainty when outcome spaces are state dependent. Theory and Decision 66(1), $1-16$.

Radzik, T. (1991). Saddle point theorems. International Journal of Game Theory 20, 23-32.

Rosenthal, R. W. (1973). A class of games possessing pure-strategy Nash equilibria. International Journal of Game Theory 2(1), 65-67.

Rosenthal, R. W. (1981). Games of perfect information, predatory pricing and the chain-store paradox. Journal of Economic Theory 25(1), 92-100.

Roth, A. E. (1977). The Shapley value as a von Neumann-Morgenstern utility. Econometrica 45(3), 657-664.

Rubinstein, A. (1991). Comments on the interpretation of game theory. Econometrica 59(4), 909-924.

Schaffer, M. E. (1988). Evolutionarily stable strategies for a finite population and a variable contest size. Journal of Theoretical Biology 132(4), 469-478.

Topkis, D. (1998). Supermodularity and Complementarity. Princeton University Press, Princeton.

Tullock, G. (1964). The irrationality of intransitivity. Oxford Economic Papers, New Series 16, 401-406.

von Neumann, J. (1928). Zur Theorie der Gesellschaftsspiele. Mathematische Annalen 100, 295-320.

von Neumann, J. and O. Morgenstern (1944). Theory of Games and Economic Behavior (1953, Third ed.). Princeton University Press, Princeton. 
Bibliography

Wald, A. (1950). Statistical decision functions. Wiley publications in statistics. Wiley, New York, NY.

Walker, M. (1977). On the existence of maximal elements. Journal of Economic Theory, 470-474. 



\section{Valorization}

Article 23 of the "Regulation governing the attainment of doctoral degrees" at Maastricht University states:

"Knowledge valorization refers to the 'process of creating value from knowledge, by making knowledge suitable and / or available for social (and / or economic) use and by making knowledge suitable for translation into competitive products, services, processes and new commercial activities' (adapted definition based on the National Valorization Committee 2011:8)."

\section{Introduction}

In this dissertation, I study certain relations between uncertainties in games and decision problems. Furthermore, I propose an alternative solution that arguably gives additional insights in some strategic decision making situations, which are not captured by the standard tools.

As I will give details below, in this dissertation I propose models that make testable and falsifiable predictions-which are indispensable for scientific research-about actual human behavior. This dissertation presents basic research rather than applied research. My findings in this dissertation are mainly relevant for (pure and applied) academic research; hence, they do not generally create immediate valorization opportunities in the short run. 


\section{Chapter 2: Two-person symmetric games and deci- sion problems}

In this chapter, I explored some interrelations between games and decision problems. I observed that some games could be framed as if a decision maker plays them against her dual-self, which led me study the links between some game and decision theoretical concepts. For example, I showed that a decision problem satisfies the von NeumannMorgenstern utility if and only if its equivalent symmetric game is a potential game. This chapters belongs to the category of basic research. Hence, its societal impact may be achieved indirectly and / or in the long run.

\section{Chapter 3: Existence of pure equilibrium in two-person symmetric zero-sum games}

In this chapter, I introduced three sufficient conditions for the existence of a pure Nash equilibrium in two-person symmetric zero-sum games including the so-called sign-quasiconcavity notion, which is a generalization of quasiconcavity in Duersch et al. (2012a). It seems that the new sufficient condition is easier to check than generalized ordinal potentials and quasiconcave games. This chapter, too, belongs to the category of basic research.

\section{Chapter 4: Optimin equilibrium: An extension of maximin strategies}

In this chapter, I developed a solution concept that extends the maximin principle to non-zero-sum games by incorporating an individual rationality constraint whereby players do not harm themselves for the sake 


\begin{tabular}{|c|c|c|c|}
\hline \multirow{3}{*}{$\begin{array}{r}\text { Rock } \\
\text { Scissors }\end{array}$} & Rock & Scissors & Paper \\
\hline & 0,0 & 1,0 & 0,1 \\
\hline & 0,1 & 0,0 & 1,0 \\
\hline & 1,0 & 0,1 & 0,0 \\
\hline
\end{tabular}

Figure 5.3: Rock-Paper-Scissors game

of harming others. A maximin strategist chooses an action to maximize the minimum utility he might receive under any conceivable play by the other player, "even assuming that his opponent is guided by the desire to inflict a loss rather than to achieve a gain" (von Neumann and Morgenstern, 1944, p. 555). Because the classical maximin strategies disregard mutual gains and losses in these interactive situations, the optimin concept better captures many economically relevant interactions that are not zero-sum.

Optimin equilibrium makes predictions about human behavior in every strategic decision making environment. Thus, it may be interesting to test the theory in various game situations. For example, Figure 5.3 illustrates the Rock-Paper-Scissors game, in which the unique optimin solution is that each player uniformly randomizes over the actions Rock, Paper, and Scissors. By doing so, he or she cannot be outguessed by his or her opponent. This prediction coincides with the prediction of Nash equilibrium - the benchmark solution concept in game theory.

It may be especially informative to test the optimin prediction in the game shown in Figure 5.4, because the unique optimin prediction differs from the unique benchmark solution, Nash equilibrium. Another game in which optimin solution differs from Nash equilibrium is the wellknown finitely repeated prisoner's dilemma. To illustrate the difference, consider the finitely repeated prisoner's dilemma with the following stage game: 


\begin{tabular}{|c|c|c|c|}
\hline & Top & Middle & Bottom \\
\hline Top & 100,100 & 100,105 & 0,0 \\
\hline Middle & 105,100 & 95,95 & 0,210 \\
\hline Bottor & 0,0 & 210,0 & 5,5 \\
\hline
\end{tabular}

Figure 5.4: A game in which the unique optimin equilibrium is (Top, Top), and the unique Nash equilibrium is (Bottom, Bottom).

\begin{tabular}{|c|c|c|}
\hline \multirow[b]{2}{*}{ Cooperate } & Cooperate & Defect \\
\hline & 3,3 & 0,5 \\
\hline Defe & 5,0 & 1,1 \\
\hline
\end{tabular}

As is well-kwown, the Tit-for-Tat strategy-in which a player begins by cooperating in the first round and then does what the opponent did in the previous round-is not a Nash equilibrium. However, it turns out to be an optimin equilibrium solution.

\section{Chapter 5: Is rationality a personal trait? A paradox}

In this chapter, I focused on the concept of rationality to answer the following question: 'Is it possible for an economic agent to be simultaneously rational in every situation she faces?' I observed that there are games and decision problems in which an agent cannot be simultaneously rational (à la von Neumann-Morgenstern) in both of them. Accordingly, I provided a unique social preference condition for an agent that makes her rational in these situations. It turns out that this condition rules out social preferences including inequality aversion and altruism.

Rationality or (expected) utility maximization has been one of the main assumptions in Economics for more than a half-century. Thus, to be able to better predict human behavior, it is important to understand the limitations of this concept in decision making situations. It would be 
Valorization

interesting to test empirically or experimentally the predictions that are provided in this chapter. 



\section{Biography}

Mehmet Ismail was born on 25 January 1987 in Kircali, Bulgaria. Then, his parents immigrated to Izmir, Turkey in 1989. After completing elementary school in Izmir, he moved to Istanbul to study at the Naval High School.

Mehmet graduated with a BSc degree in Economics at Y1ldız Technical University in 2010. Then he obtained Master 1 and Master 2 degrees in Applied Mathematics at the University of Paris 1 Panthéon-Sorbonne in 2011 and 2012, respectively, and a joint MSc degree in Quantitative Economics at the University of Paris 1 and Bielefeld University in 2012 as part of the European Union's QEM Erasmus Mundus programme.

In 2012, Mehmet joined Maastricht University as a PhD candidate in Economics under the supervision of Jean-Jacques Herings and Ronald Peeters. During his studies, he made research visits to Warwick University (one month), and New York University (four months). Some of the results of his work as a PhD student are presented in this dissertation.

The paper, Making the Rules of Sports Fairer, that Mehmet co-authored with Steven Brams not only is forthcoming at SIAM Review, but it has garnered considerable media coverage including in the Daily Mail, The Telegraph, The Sun, BBC Radio 5 Live, and De Limburger. The Catch-Up game that he co-authored with Aaron Isaksen, Steven Brams, and Andy Nealen, was copyrighted by New York University (75\%) and Maastricht University (25\%). 
